\title{
The Optimal Set and Optimal Partition Approach to Linear and Quadratic Programming*
}

\author{
Arjan B. Berkelaar, Kees Roos and Tamás Terlaky \\ October 16, 1996 \\ Department of Econometrics and Operations Research \\ Econometric Institute \\ Faculty of Economics \\ Erasmus University Rotterdam \\ P.O.Box 1738, 3000 DR Rotterdam, The Netherlands.
}

\begin{abstract}
In this chapter we describe the optimal set approach for sensitivity analysis for LP. We show that optimal partitions and optimal sets remain constant between two consecutive transition-points of the optimal value function. The advantage of using this approach instead of the classical approach (using optimal bases) is shown. Moreover, we present an algorithm to compute the partitions, optimal sets and the optimal value function. This is a new algorithm and uses primal and dual optimal solutions. We also extend some of the results to parametric quadratic programming, and discuss differences and resemblances with the linear programming case.
\end{abstract}

Key words: Sensitivity Analysis, Parametric Programming, Complementarity

*This paper will appear as Chapter 6 in H. Greenberg and T. Gal (editors), Recent Advances in Sensitivity Analysis and Parametric Programming, Kluwer Publishers, (1997). 


\section{Introduction}

In this chapter we deal with parametric versions of linear programming (LP) and convex quadratic programming (QP). First consider the LP problem $(P)$ in standard format

$$
\text { (P) } \min \left\{c^{T} x: A x=b, x \geq 0\right\},
$$

where $c, x \in \mathbb{R}^{n}, b \in \mathbb{R}^{m}$, and $A$ is an $m \times n$ matrix with full row rank. The dual problem ( $\left.D\right)$ is written as

$$
\max \left\{b^{T} y: A^{T} y+s=c, s \geq 0\right\},
$$

where $y \in \mathbb{R}^{m}$ and $s \in \mathbb{R}^{n}$. The input data for both problems consists of the matrix $A$, and the vectors $b$ and $c$.

In Chapter 4 it is shown that difficulties may arise, when the problem under consideration is degenerate. It is stated that the optimal basis may not be unique, that multiple optimal solutions may exist, and that the notion of shadow price is not correctly defined ${ }^{1}$. In Chapter 3 strictly complementary solutions are already mentioned. These solutions, although already shown to exist in 1956 by Goldman and Tucker [10], came into view in the 1980s due to the immense popularity of interior point methods (Den Hertog [14] and Jansen [15]). This popularity was initiated by the seminal paper of Karmarkar [17]. Güler and Ye [12] showed that interior point methods for LP generate a strictly complementary solution (in the limit). This triggered interest in investigating parametric analysis without using bases, instead making use of strictly complementary solutions (Adler and Monteiro [1], Mehrotra and Monteiro [22], and Jansen et al. [16]). In this chapter we describe this approach and formulate a new algorithm to compute the optimal value function, which uses primal and dual optimal solutions.

Let us now turn to the quadratic programming formulation. The general CQP problem is given by

$$
(Q P) \quad \min \left\{c^{T} x+\frac{1}{2} x^{T} Q x: A x=b, \geq 0\right\},
$$

where $c, x \in \mathbb{R}^{n}, b \in \mathbb{R}^{m}, A$ an $m \times n$ matrix with full row rank and $Q$ a symmetric positive semidefinite $n \times n$ matrix. The Wolfe-dual of $(Q P)$ is given by

$$
(Q D) \quad \max \left\{b^{T} y-\frac{1}{2} u^{T} Q u: A^{T} y+s-Q u=c, s \geq 0\right\},
$$

where $y \in \mathbb{R}^{m}$ and $u, s \in \mathbb{R}^{n}$. The input data for both problems consists of the matrix $Q$, the matrix $A$, and the vectors $b$ and $c$. We assume that $Q$ is positive semidefinite resulting in a convex quadratic programming problem. Unless stated otherwise QP refers to convex quadratic programming problem. It is well known that if there exist optimal solutions for (QP), then there also exist optimal solutions for which $x=u$. Furthermore, it is clear that LP is a special case of $\mathrm{QP}^{2}$. In this chapter we are only concerned with changes in the vectors $b$ and $c, A$ and $Q$ are taken to be fixed.

Although sensitivity analysis and parametric programming for QP are not being performed on a large scale, there is at least one important application on a commercial level. The well-known Markowitz mean-variance model [20] for modern portfolio theory is formulated as a parametric QP. The optimal value function of this parametric QP is known as the efficient frontier. The efficient frontier is a useful tool, being used by various different financial institutions for portfolio decision problems. Recent studies on computing the efficient frontier (see, e.g. [19, 26, 27]) all use optimal bases. Similar difficulties w.r.t. degeneracy as in the LP case exist for QP (the optimal basis ${ }^{3}$

\footnotetext{
${ }^{1}$ See also, Gal [9] and Greenberg [11] for a survey and Rubin and Wagner [24] for an overview of practical implications.

${ }^{2}$ Take $Q$ to be the zero matrix.

${ }^{3}$ Note that the primal or the dual QP problem may have a solution that is not a basic solution. However if we consider both the primal and the dual QP problem together, then, if the problem has a solution there always exists an optimal basis (rewrite the primal and dual problem as a linear complementarity problem to see this). In this chapter we take the freedom to speak about an optimal basis in the latter sense.
} 
may not be unique, multiple solutions may exist, etc.). Berkelaar et al. [4] consider the efficient frontier as the outcome of their analysis for parametric QP using maximal complementary solutions. Interior point methods for QP generate such a maximal complementary solution (in the limit). A related result to this was already shown by McLinden [21] in 1980 (see also Güler and Ye [13]). We describe parametric analysis for QP using maximal complementary solutions in this chapter, and again formulate an algorithm to compute the optimal value function. This algorithm uses both primal and dual solutions and their supports.

Let us denote the optimal value of $(Q P)$ and $(Q D)$ as $z(b, c)$, with $z(b, c)=-\infty$ if $(Q P)$ is unbounded and $(Q D)$ infeasible and $z(b, c)=\infty$ if $(Q D)$ is unbounded and $(Q P)$ infeasible. If $(Q P)$ and $(Q D)$ are both infeasible then $z(b, c)$ is undefined. We call $z$ the optimal value function for the data $b$ and $c$. Since LP is a special case of QP, we also denote the optimal value function for LP problems by $z(b, c)$ with the same conventions.

Although in the literature assumptions are often made to prevent situations concerning degeneracy, we shall not do so here. The main tools we use are the existence of strictly complementary and maximal complementary solutions in LP and QP respectively. Such solutions uniquely define the partition of the problem. We show that the pieces of the optimal value function correspond to intervals for the parameter on which the partition is constant. The proposed algorithms to compute the optimal value function are based on this key result.

This chapter is organized as follows. In Section 2 we consider a transportation (LP) example to show that the classical approach to sensitivity analysis based on optimal bases leads to difficulties in case of degeneracy. Section 3 describes the optimal partition and we show how this concept and given optimal solutions can be used to characterize the optimal sets of LP and QP problems. In Section 4 we consider parametric LP based on the optimal set approach. We omit proofs in Section 4, and postpone them to Section 5. In Section 5 we consider parametric QP. Since LP is a special case of QP it is left to the reader to specialize the proofs to LP. Some results for LP and their proofs can be formulated and presented differently or need no proof at all. Section 4 has been organized so as to concentrate on the resemblances between parametric LP and parametric QP. For a more detailed analysis in LP the reader is referred to Jansen et al. [15, 16]. Finally we close this chapter by outlining how the ideas of Sections 4 and 5 can be applied to sensitivity analysis.

\section{The Optimal Bases Approach - An Example}

In commercial packages for LP and QP usually the possibility is offered to perform sensitivity analysis. As far as we know sensitivity analysis in all existing commercial packages is based on optimal bases. As a result, the outcome of the sensitivity analysis is often only partly correct. In this section we show this using an example.

The classical approach to sensitivity analysis is based on pivoting methods (such as the Simplex method for LP) for solving LP and QP problems. These methods produce a so-called basic solution

of the problem. It suffices for our purpose to know that such a solution is determined by an optimal basis. We only briefly consider a small textbook LP problem to illustrate problems that can occur in case of degeneracy. This example is taken from Jansen [15]. For a more detailed description we refer to Jansen et al. [16] and Jansen [15]. To illustrate the shortcomings of the implemented sensitivity analysis techniques we apply several commercial packages to a small LP problem.

\subsection{Comparison of the classical and the new approach}

Example 1 We consider a simple transportation problem with three supply and demand nodes. 
Table 1: Sensitivity analysis for a transportation problem - RHS changes

\begin{tabular}{|l|c|c|c|c|c|c|}
\hline \multirow{2}{*}{ LP-package } & \multicolumn{7}{|c|}{ Ranges of supply and demand values } \\
\cline { 2 - 7 } & $b_{1}(2)$ & $b_{2}(6)$ & $b_{3}(5)$ & $b_{4}(3)$ & $b_{5}(3)$ & $b_{6}(3)$ \\
\hline CPLEX & {$[0,3]$} & {$[4,7]$} & {$[1, \infty)$} & {$[2,7]$} & {$[2,5]$} & {$[2,5]$} \\
LINDO & {$[1,3]$} & {$[2, \infty)$} & {$[4,7]$} & {$[2,4]$} & {$[1,4]$} & {$[1,7]$} \\
PC-PROG & {$[0, \infty)$} & {$[4, \infty)$} & {$[3,6]$} & {$[2,5]$} & {$[0,5]$} & {$[2,5]$} \\
XMP & {$[0,3]$} & {$[6,7]$} & {$[1, \infty)$} & {$[2,3]$} & {$[2,3]$} & {$[2,7]$} \\
OSL & {$[0,3]$} & {$[4,7]$} & $(-\infty, \infty)$ & {$[2,7]$} & {$[2,5]$} & {$[2,5]$} \\
\hline Correct range & {$[0, \infty)$} & {$[2, \infty)$} & {$[1, \infty)$} & {$[0,7]$} & {$[0,7]$} & {$[0,7]$} \\
\hline
\end{tabular}

$$
\begin{array}{ll}
\min & \sum_{i=1}^{9} x_{i} \\
\text { s.t. } & x_{1}+x_{2}+x_{3}+x_{10}=2 \\
& x_{4}+x_{5}+x_{6}+x_{11}=6 \\
x_{7}+x_{8}+x_{9}+x_{12}=5 \\
x_{1}+x_{4}+x_{7}-x_{13}=3 \\
x_{2}+x_{5}+x_{8}-x_{14}=3 \\
x_{3}+x_{6}+x_{9}-x_{15}=3 \\
x_{i} \geq 0, i=1, \ldots, 15 .
\end{array}
$$

The results of a sensitivity analysis are shown in Table 1 . The columns correspond to the RHS elements. The rows in the table correspond to five packages CPLEX, LINDO, PC-PROG, XMP and OSL and show the ranges produced by these packages. The last row contains the ranges calculated by the approach outlined in this chapter ${ }^{4}$.

The different ranges in the Table 1 are due to the different optimal bases that are found by the different packages. For each optimal basis the range can be calculated by examining for which values of the RHS element the optimal basis remains constant. The table demonstrates the weaknesses of the optimal bases approach that is implemented in the commercial packages. Sensitivity analysis is considered to be a tool for obtaining information about the bottlenecks and degrees of freedom in the problem. The information provided by the commercial packages is confusing and hardly allows a solid interpretation. The difficulties lie in the fact that an optimal basis need not be unique.

In this chapter we show that the optimal partition of a strictly complementary solution for LP or a maximal complementary solution for QP leads to a much more solid analysis. The reason for this is that the optimal partition is unique for any strictly or maximal complementary solution.

\section{Optimal Partitions and Optimal Sets}

\subsection{Linear Programming}

The feasible regions of $(P)$ and $(D)$ are denoted as

$$
\begin{aligned}
& \mathcal{P}:=\{x: A x=b, x \geq 0\}, \\
& \mathcal{D}:=\left\{(y, s): A^{T} y+s=c, s \geq 0\right\} .
\end{aligned}
$$

\footnotetext{
${ }^{4}$ The range provided by the IBM package OSL (Optimization Subroutine Library) for $b_{3}$ is not a subrange of the correct range; this must be due to a bug in OSL. The correct range for the optimal basis found by OSL should be $[1, \infty)$.
} 
Assuming that $(P)$ and $(D)$ are both feasible, the optimal sets of $(P)$ and $(D)$ are denoted as $\mathcal{P}^{*}$ and $\mathcal{D}^{*}$. We define the index sets $B$ and $N$ by

$$
\begin{aligned}
B & :=\left\{i: x_{i}>0 \text { for some } x \in \mathcal{P}^{*}\right\} \\
N & :=\left\{i: s_{i}>0 \text { for some }(y, s) \in \mathcal{D}^{*}\right\} .
\end{aligned}
$$

The Duality Theorem for LP implies that $B \cap N=\phi$, and the Goldman and Tucker Theorem [10] that

$$
B \cup N=\{1,2, \cdots, n\} .
$$

So, $B$ and $N$ form a partition of the full index set. This (ordered) partition, denoted as $\pi=(B, N)$, is the optimal partition of the problem $(P)$ and of the problem $(D)$.

In the rest of this chapter we assume that $b$ and $c$ are such that $(P)$ and $(D)$ have optimal solutions, and $\pi=(B, N)$ denotes the optimal partition of both problems. By definition, the optimal partition is determined by the set of optimal solutions for $(P)$ and $(D)$. In this section it is made clear that, conversely, the optimal partition provides essential information on the optimal solution sets $\mathcal{P}^{*}$ and $\mathcal{D}^{*}$.

We use the notation $x_{B}$ and $x_{N}$ to refer to the restriction of a vector $x \in \mathbb{R}^{n}$ to the coordinate sets $B$ and $N$ respectively. Similarly, $A_{B}$ denotes the restriction of $A$ to the columns in $B$, and $A_{N}$ the restriction of $A$ to the columns in $N$. Now the sets $\mathcal{P}^{*}$ and $\mathcal{D}^{*}$ can be described in terms of the optimal partition. The next lemma follows immediately from the Duality Theorem and the definition of the optimal partition for LP and is therefore stated without proof.

Lemma 1 Let $x^{*} \in \mathcal{P}^{*}$ and $\left(y^{*}, s^{*}\right) \in \mathcal{D}^{*}$. Given the optimal partition $(B, N)$ of $(P)$ and $(D)$, the optimal sets of both problems are given by

$$
\begin{aligned}
\mathcal{P}^{*} & =\left\{x: x \in \mathcal{P}, x^{T} s^{*}=0\right\} \\
& =\left\{x: x \in \mathcal{P}, x_{N}=0\right\}, \\
\mathcal{D}^{*} & =\left\{(y, s):(y, s) \in \mathcal{D}, s^{T} x^{*}=0\right\} \\
& =\left\{(y, s):(y, s) \in \mathcal{D}, s_{B}=0\right\} .
\end{aligned}
$$

The next result deals with the dimensions of the optimal sets of $(P)$ and $(D)$. Here, as usual the (affine) dimension of a subset of $\mathbb{R}^{k}$ is the dimension of the smallest affine subspace in $\mathbb{R}^{k}$ containing the subset.

Lemma 2 One has

$$
\begin{aligned}
\operatorname{dim} \mathcal{P}^{*} & =|B|-\operatorname{rank}\left(A_{B}\right) \\
\operatorname{dim} \mathcal{D}^{*} & =m-\operatorname{rank}\left(A_{B}\right)
\end{aligned}
$$

Lemma 2 immediately implies that $(P)$ has a unique solution ${ }^{5}$ if and only if $\operatorname{rank}\left(A_{B}\right)=|B|$. Clearly this happens if and only if the columns in $A_{B}$ are linearly independent. Also, $(D)$ has a unique solution if and only if $\operatorname{rank}\left(A_{B}\right)=m$, which happens if and only if the rows in $A_{B}$ are linearly independent. Thus, both (P) and (D) have a unique solution if and only if $A_{B}$ is a basis (the unique optimal basis).

\footnotetext{
${ }^{5}$ Notice that we speak of uniqueness of the optimal solution and not about optimal basis (which is not necessarily unique).
} 


\subsection{Quadratic Programming}

In this section we describe analogous results, as in the preceding section, for QP. The proofs of these results are included here and it is left to the reader to specialize these proofs to LP. The feasible regions of $(Q P)$ and $(Q D)$ are denoted as

$$
\begin{aligned}
\mathcal{Q P} & :=\{x: A x=b, x \geq 0\} \\
\mathcal{Q D} & :=\left\{(u, y, s): A^{T} y+s-Q u=c, s \geq 0\right\} .
\end{aligned}
$$

We start with the Duality Theorem for QP, which is stated without proof (see, e.g. Dorn [8]).

Theorem 3 If $x$ is feasible for $(Q P)$ and $(u, y, s)$ for $(Q D)$, then these solutions are optimal if and only if $Q x=Q u$ and $x^{T} s=0$.

Assuming that $(Q P)$ and $(Q D)$ are both feasible, the optimal sets of $(Q P)$ and $(Q D)$ are denoted as $\mathcal{Q} \mathcal{P}^{*}$ and $\mathcal{Q D} \mathcal{D}^{*}$. These optimal sets can be characterized by maximal complementary solutions and the corresponding partition (Güler and Ye [13]). Let us define

$$
\begin{aligned}
B & :=\left\{i: x_{i}>0 \text { for some } x \text { of } \mathcal{Q P} \mathcal{P}^{*}\right\}, \\
N & :=\left\{i: s_{i}>0 \text { for some }(u, y, s) \text { of } \mathcal{Q} \mathcal{D}^{*}\right\}, \\
T & :=\{1, \ldots, n\} \backslash(B \cup N) .
\end{aligned}
$$

The Duality Theorem for QP implies that $B \cap N=\phi$. Note that, contrary to LP, in QP the Goldman and Tucker Theorem [10] does not hold, $T$ may be nonempty. So $B, N$ and $T$ form a partition of the full index set. This (ordered) partition, denoted as $\pi=(B, N, T)$, is the optimal partition of the problem $(Q P)$ and of the problem $(Q D)$. A maximal complementary solution $(x, y, s)$ is a solution for which

$$
x_{i}>0 \Longleftrightarrow i \in B, \quad s_{i}>0 \Longleftrightarrow i \in N .
$$

The existence of such a solution is a consequence of the convexity of the optimal sets of $(Q P)$ and $(Q D)$ and was introduced by McLinden [21]. He showed an important result concerning such solutions, which was used by Güler and Ye [13] to show that interior point methods (as Anstreicher et al. [2], Carpenter et al. [5] and Vanderbei [25]) generate such a solution (in the limit).

In Chapter 3 the definition of the support of a vector was given. In this chapter we denote the support of a vector $v$ by $\sigma(v)$. Hence, given a strictly complementary or maximal complementary solution $\left(x^{*}, y^{*}, s^{*}\right)$ we have $B=\sigma\left(x^{*}\right)$ and $N=\sigma\left(s^{*}\right)$.

In the rest of this chapter we assume that $b$ and $c$ are such that $(Q P)$ and $(Q D)$ have optimal solutions, and $\pi=(B, N, T)$ denote the optimal partition of both problems. By definition, the optimal partition is determined by the set of optimal solutions for $(Q P)$ and $(Q D)$. In this section we show that, conversely, the optimal partition provides essential information on the optimal solution $\operatorname{sets} \mathcal{Q} \mathcal{P}^{*}$ and $\mathcal{Q} \mathcal{D}^{*}$

In the introduction we already mentioned that there exist optimal solutions for which $x=u$; when useful we denote a dual optimal solution by $(x, y, s)$ instead of $(u, y, s)$. Later we also use the following well-known result.

Lemma 4 Let $\left(x^{*},\left(u^{*}, y^{*}, s^{*}\right)\right)$ and $(\tilde{x},(\tilde{u}, \tilde{y}, \tilde{s}))$ both be optimal solutions of $(Q P)$ and $(Q D)$. Then $Q x^{*}=Q \tilde{x}=Q u^{*}=Q \tilde{u}, c^{T} x^{*}=c^{T} \tilde{x}$ and $b^{T} y^{*}=b^{T} \tilde{y}$.

Proof: Let us first consider the case where $x^{*}=u^{*}$ and $\tilde{x}=\tilde{u}$. Since $\left(x^{*}, y^{*}, s^{*}\right)$ and $(\tilde{x}, \tilde{y}, \tilde{s})$ are both optimal, we conclude that

$$
c^{T} x^{*}+\frac{1}{2} x^{*} Q x^{*}=b^{T} \tilde{y}-\frac{1}{2} \tilde{x}^{T} Q \tilde{x}
$$


Using that $A^{T} \tilde{y}+\tilde{s}-Q \tilde{x}=c$ and multiplying by $\left(x^{*}\right)^{T}$ it follows,

$$
\frac{1}{2}\left(x^{*} Q x^{*}+\tilde{x} Q \tilde{x}-2 \tilde{x} Q x^{*}\right)=b^{T} \tilde{y}-c^{T} x^{*}-\tilde{x} Q x^{*}=0 .
$$

Thus, $\left(x^{*}-\tilde{x}\right)^{T} Q\left(x^{*}-\tilde{x}\right)=0$, which implies $Q\left(x^{*}-\tilde{x}\right)=0$, i.e. $Q x^{*}=Q \tilde{x}$, since $Q$ is positive semidefinite. Using that $Q x^{*}=Q \tilde{x}$ we conclude that $c^{T} x^{*}=c^{T} \tilde{x}$ and $b^{T} y^{*}=b^{T} \tilde{y}$. From the Duality Theorem the proof is completed.

We use the notation $x_{B}, x_{N}$ and $x_{T}$ to refer to the restriction of a vector $x \in \mathbb{R}^{n}$ to the coordinate sets $B, N$ and $T$ respectively. Similarly, $A_{B}$ denotes the restriction of $A$ to the columns in $B, A_{N}$ the restriction of $A$ to the columns in $N$, and $A_{T}$ the restriction of $A$ to the columns in $T$. The matrix $Q$ is partitioned similarly. Now the sets $\mathcal{Q P} \mathcal{P}^{*}$ and $\mathcal{Q} \mathcal{D}^{*}$ can be described in terms of the optimal partition. The next lemma follows immediately from the Duality Theorem and the definition of the optimal partition for QP and is therefore stated without proof.

Lemma 5 Let $x^{*} \in \mathcal{Q P} \mathcal{P}^{*}$ and $\left(u^{*}, y^{*}, s^{*}\right) \in \mathcal{Q D}^{*}$. Given the optimal partition $(B, N, T)$ of $(Q P)$ and $(Q D)$, the optimal sets of both problems are given by

$$
\begin{aligned}
\mathcal{Q P} & =\left\{x: x \in \mathcal{Q P}, x^{T} s^{*}=0, Q x=Q u^{*}\right\} \\
& =\left\{x: x \in \mathcal{Q P}, x_{N \cup T}=0, Q x=Q u^{*}\right\}, \\
\mathcal{Q D}^{*} & =\left\{(u, y, s):(u, y, s) \in \mathcal{Q D}, s^{T} x^{*}=0, Q u=Q x^{*}\right\} \\
& =\left\{(u, y, s):(u, y, s) \in \mathcal{Q D}, s_{B \cup T}=0, Q u=Q x^{*}\right\} .
\end{aligned}
$$

Lemma 6 Let $M \in \mathbb{R}^{n \times n}$ be a symmetric positive semidefinite matrix, partitioned according to $B, N$ and $T$. Then

$$
\mathcal{N}\left(M_{B B}\right)=\mathcal{N}\left(M_{B}\right)
$$

Proof: $\quad$ Let $x$ be an arbitrary vector in $\mathbb{R}^{n}$ and partitioned according to $B$ and $\bar{B}=N \cup T$. Then

$$
x^{T} M x=x_{B}^{T} M_{B B} x_{B}+2 x_{\bar{B}}^{T} M_{\bar{B} B} x_{B}+x_{\bar{B}}^{T} M_{\bar{B} \bar{B}} x_{\bar{B}} .
$$

The result is proven by contradiction. Suppose to the contrary that $\mathcal{N}\left(M_{B B}\right) \neq \mathcal{N}\left(M_{\cdot_{B}}\right)$. Take $x_{B}$ from the null space of $M_{B B}$. Then, $M_{B B} x_{B}=0$ and $M_{\bar{B} B} x_{B} \neq 0$. Let $\varepsilon$ be given and consider $x=\varepsilon \bar{x}$. Furthermore let $\left.\alpha=x_{\bar{B}}^{T}\left(2 M_{\bar{B} B} x_{B}\right)\right)$ and $\beta=x_{\bar{B}}^{T} M_{\bar{B} \bar{B}} x_{\bar{B}}$. It can easily be seen that $\alpha \neq 0$ and $\beta \geq 0$. Now we can rewrite $x^{T} M x$ as follows

$$
x^{T} M x=\varepsilon\left(x_{\bar{B}}^{T}\left(2 M_{\bar{B} B} \bar{x}_{B}\right)\right)+\varepsilon^{2}\left(\bar{x}_{\bar{B}}^{T} M_{\bar{B} \bar{B}} \bar{x}_{\bar{B}}\right)=\varepsilon \alpha+\varepsilon^{2} \beta .
$$

Thus, $x^{T} M x<0$ if and only if $\varepsilon<\frac{-\alpha}{\beta}$. Since $M$ is positive semidefinite we have a contradiction. This implies the result.

The next result deals with the dimensions of the optimal sets of $(Q P)$ and $(Q D)$.

Lemma 7 One has

$$
\begin{aligned}
\operatorname{dim} \mathcal{Q} \mathcal{P}^{*} & =|B|-\operatorname{rank}\left(\begin{array}{c}
A_{B} \\
Q_{B B}
\end{array}\right) \\
\operatorname{dim} \mathcal{Q} \mathcal{D}^{*} & =m-\operatorname{rank}\left(\begin{array}{c}
A_{B}^{T} \\
A_{T}^{T}
\end{array}\right)+n-\operatorname{rank}(Q) .
\end{aligned}
$$


Proof: Let a dual optimal solution $u$ be given. Then, by Lemma 5 the optimal set of $(Q P)$ is given by

$$
\mathcal{Q P} \mathcal{P}^{*}=\left\{x: A x=b, x_{B} \geq 0, x_{N \cup T}=0, Q x=Q u\right\},
$$

and hence the smallest affine subspace of $\mathbb{R}^{n}$ containing $\mathcal{Q P} \mathcal{P}^{*}$ is given by

$$
\left\{x: A_{B} x_{B}=b, x_{N \cup T}=0, Q \cdot{ }_{B} x_{B}=Q u\right\} .
$$

The dimension of this affine space is equal to the dimension of the null space of $\left(A_{B}^{T} Q_{{ }_{B}}^{T}\right)^{T}$. Since the dimension of the null space of this matrix is given by $|B|-\operatorname{rank}\left(\left(A_{B}^{T} Q_{\cdot}^{T}\right)^{T}\right)$, the first statement follows from Lemma 6.

For the proof of the second statement we use that the dual optimal set can be described as in Lemma 5 . Let $x$ be a given primal solution. Then the optimal dual set is given by

$$
\mathcal{Q D}^{*}=\left\{(u, y, s): A^{T} y+s-Q u=c, s_{B \cup T}=0, s_{N} \geq 0, Q u=Q x\right\} .
$$

This is equivalent to

$$
\begin{aligned}
\mathcal{Q D}^{*}=\{(u, y, s): & A_{B}^{T} y-(Q u)_{B}=c_{B}, \\
& A_{T}^{T} y-(Q u)_{T}=c_{T}, \\
& A_{N}^{T} y+s_{N}-(Q u)_{N}=c_{N}, \\
& \left.s_{B}=0, s_{T}=0, s_{N} \geq 0, Q u=Q x\right\} .
\end{aligned}
$$

The smallest affine subspace containing this set is

$$
\begin{aligned}
\mathcal{Q D}^{*}=\{(u, y, s): & A_{B}^{T} y-(Q u)_{B}=c_{B}, \\
& A_{T}^{T} y-(Q u)_{T}=c_{T}, \\
& A_{N}^{T} y+s_{N}-(Q u)_{N}=c_{N}, \\
& \left.s_{B}=0, s_{T}=0, Q u=Q x\right\} .
\end{aligned}
$$

Obviously $s_{N}$ is uniquely determined by $y$, and any $y$ satisfying $A_{B}^{T} y-(Q u)_{B}=c_{B}, A_{T}^{T} y-(Q u)_{T}=$ $c_{T}$ yields a point in the affine $(y, s)$-space. Hence the dimension of this affine space is equal to the dimension of the null space of $\left(A_{B} A_{T}\right)^{T}$. The dimension of the null space of this matrix equals $m-\operatorname{rank}\left(\left(A_{B} A_{T}\right)^{T}\right)$. Furthermore, any $u$ satisfying $Q u=Q x$ yields a point in the affine $u$-space. Hence the dimension of this affine space is equal to the dimension of the null space of $Q$, which equals $n-\operatorname{rank}(Q)$. Combining these results completes the proof.

Note that when $Q=0$ is substituted in the formulae of Lemma 7 the dimension of the dual set is not equal to the dimension in Lemma 2. Nevertheless, the results are consistent since $u$ is not to be taken into account in the formula for the dimension of the dual optimal set.

In this section we have characterized the optimal sets in LP and QP by using the optimal partition. In LP there is a one to one relation between the optimal partition and the primal and dual optimal sets. Given an optimal partition for an LP problem, the primal and dual optimal sets can be characterized. In QP we need the optimal partition and a dual optimal solution to characterize the primal optimal set; and the optimal partition and a primal optimal solution to characterize the dual optimal set. The results for parametric LP are thus obtained from parametric QP using the characterization of the optimal sets by the optimal partition.

\section{Parametric Linear Programming}

In this section we investigate the effects of changes in the vectors $b$ and $c$ on the optimal value $z(b, c)$ of $(P)$ and $(D)$. We consider one-dimensional parametric perturbations of $b$ and $c$. So we want to study

$$
z(b+\lambda \Delta b, c+\mu \Delta c)
$$


as a function of the parameters $\lambda$ and $\mu$, where $\Delta b$ and $\Delta c$ are given perturbation vectors. So, the vectors $b$ and $c$ are fixed, and the variations come from the parameters $\lambda$ and $\mu$. We denote the perturbed problems as $\left(P_{\lambda}\right)$ and $\left(D_{\mu}\right)$, and their feasible regions as $\mathcal{P}_{\lambda}$ and $\mathcal{D}_{\mu}$ respectively. The dual problem of $\left(P_{\lambda}\right)$ is denoted as $\left(D_{\lambda}\right)$ and the dual problem of $\left(D_{\mu}\right)$ is denoted as $\left(P_{\mu}\right)$. Observe that the feasible region of $\left(D_{\lambda}\right)$ is simply $\mathcal{D}$ and the feasible region of $\left(P_{\mu}\right)$ is simply $\mathcal{P}$. We use the superscript * to refer to the optimal set of each of these problems. As we already mentioned in the introduction we postpone proofs that have an analogue in QP to the next section, where we consider parametric convex quadratic programming.

We assume that $b$ and $c$ are such that $(P)$ and $(D)$ are both feasible. Then $z(b, c)$ is well defined and finite. It is convenient to introduce the following notations:

$$
\begin{gathered}
b(\lambda):=b+\lambda \Delta b, \quad c(\mu):=c+\mu \Delta c, \\
f(\lambda):=z(b(\lambda), c), \quad g(\mu):=z(b, c(\mu)) .
\end{gathered}
$$

Here the domain of the parameters $\lambda$ and $\mu$ is taken as large as possible. Let us consider the domain of $f$. The function $f$ is defined as long as $z(b(\lambda), c)$ is well defined. Since the feasible region of $\left(D_{\lambda}\right)$ is constant when $\lambda$ varies, and since we assumed that $\left(D_{\lambda}\right)$ is feasible for $\lambda=0$, it follows that $\left(D_{\lambda}\right)$ is feasible for all values of $\lambda$. Therefore, since $f(\lambda)$ is well defined if the dual problem $\left(D_{\lambda}\right)$ has an optimal solution and $f(\lambda)$ is not defined (or infinity) if the dual problem $\left(D_{\lambda}\right)$ is unbounded. Using the Duality Theorem it follows that $f(\lambda)$ is well defined if and only if the primal problem $\left(P_{\lambda}\right)$ is feasible. In exactly the same way it can be understood that the domain of $g$ consists of all $\mu$ for which $\left(D_{\mu}\right)$ is feasible (and $\left(P_{\mu}\right)$ bounded). The following theorem is well known.

Theorem 8 The domains of $f$ and $g$ are closed intervals on the real line.

\subsection{Optimal value function and optimal sets on a linearity interval}

In this section we show that the functions $f(\lambda)$ and $g(\mu)$ are piecewise linear on their domains. The pieces correspond to intervals where the partition is constant. For any $\lambda$ in the domain of $f$ we denote the optimal set of $\left(P_{\lambda}\right)$ as $\mathcal{P}_{\lambda}^{*}$ and the optimal set of $\left(D_{\lambda}\right)$ as $\mathcal{D}_{\lambda}^{*}$. The results in this section are related to similar results, already obtained in 1960 s by e.g. Bereanu [3], Charnes and Cooper [6], and Kelly [18] (see also Dinkelbach [7, Chapter 5, Sections 1 and 2] and Gal [9]). The first theorem shows that the dual optimal set is constant on certain intervals and that $f$ is linear on these intervals. This results from the fact that the optimal partition is constant on certain intervals (see the next section) and the characterization of the optimal sets (see Lemma 1).

Theorem 9 Let $\lambda_{1}$ and $\lambda_{2}>\lambda_{1}$ be such that $\mathcal{D}_{\lambda_{1}}^{*}=\mathcal{D}_{\lambda_{2}}^{*}$. Then $\mathcal{D}_{\lambda}^{*}$ is constant for all $\lambda \in\left[\lambda_{1}, \lambda_{2}\right]$ and $f(\lambda)$ is linear on the interval $\left[\lambda_{1}, \lambda_{2}\right]$.

From this theorem we conclude the following result giving a partition of the domain of $f$ in intervals on which the dual optimal set remains constant.

Theorem 10 The domain of $f$ can be partitioned in a finite set of subintervals such that the dual optimal set is constant on a subinterval.

Using the former two theorems we conclude that $f$ is convex and piecewise linear.

Theorem 11 The optimal value function $f(\lambda)$ is continuous, convex and piecewise linear.

The values of $\lambda$ where the partition of the optimal value function $f(\lambda)$ changes are called transitionpoints of $f$, and any interval between two successive transition-points of $f$ is called a linearity interval of $f$. In a similar way we define transition-points and linearity intervals for $g$. Each of the above results on $f(\lambda)$ has its analogue for $g(\mu)$. 
Theorem 12 Let $\mu_{1}$ and $\mu_{2}>\mu_{1}$ be such that $\mathcal{P}_{\mu_{1}}^{*}=\mathcal{P}_{\mu_{2}}^{*}$. Then $\mathcal{P}_{\mu}^{*}$ is constant for all $\mu \in\left[\mu_{1}, \mu_{2}\right]$ and $g(\mu)$ is linear on the interval $\left[\mu_{1}, \mu_{2}\right]$.

Theorem 13 The domain of $g$ can be partitioned in a finite set of subintervals such that the primal optimal set is constant on a subinterval.

Theorem 14 The optimal value function $g(\mu)$ is continuous, concave and piecewise linear.

\subsection{Extreme points of a linearity interval}

In this section we assume that $\bar{\lambda}$ belongs to the interior of a linearity interval $\left[\lambda_{1}, \lambda_{2}\right]$. Given an optimal solution of $\left(D_{\bar{\lambda}}\right)$ we show how the extreme points $\lambda_{1}$ and $\lambda_{2}$ of the linearity interval containing $\bar{\lambda}$ can be found by solving two auxiliary linear optimization problems. This is stated in the next theorem.

Theorem 15 Let $\bar{\lambda}$ be arbitrary and let $\left(y^{*}, s^{*}\right)$ be any optimal solution of $\left(D_{\bar{\lambda}}\right)$. Then the extreme points of the linearity interval $\left[\lambda_{1}, \lambda_{2}\right]$ containing $\bar{\lambda}$ follow from

$$
\begin{aligned}
& \lambda_{1}=\min _{\lambda, x}\left\{\lambda: A x=b+\lambda \Delta b, x \geq 0, x^{T} s^{*}=0\right\} \\
& \lambda_{2}=\max _{\lambda, x}\left\{\lambda: A x=b+\lambda \Delta b, x \geq 0, x^{T} s^{*}=0\right\} .
\end{aligned}
$$

Theorem 16 Let $\bar{\lambda}$ be a transition-point and let $\left(y^{*}, s^{*}\right)$ be a strictly complementary optimal solution of $\left(D_{\bar{\lambda}}\right)$. Then the numbers $\lambda_{1}$ and $\lambda_{2}$ given by Theorem 15 satisfy $\lambda_{1}=\lambda_{2}=\bar{\lambda}$.

Proof: If $\left(y^{*}, s^{*}\right)$ is a strictly complementary optimal solution of $\left(\mathrm{D}_{\bar{\lambda}}\right)$ then it uniquely determines the optimal partition of $\left(\mathrm{D}_{\bar{\lambda}}\right)$ and this partition differs from the optimal partition corresponding to the optimal sets at the linearity intervals surrounding $\bar{\lambda}$. Hence $\left(y^{*}, s^{*}\right)$ does not belong to the optimal sets at the linearity intervals surrounding $\bar{\lambda}$. Furthermore it holds in any transition-point that that $\Delta b^{T} y^{*}$ satisfies

$$
\Delta b^{T} y^{-}<\Delta b^{T} y^{*}<\Delta b^{T} y^{+}
$$

where $y^{-}$belongs to the linearity interval just to the left of $y$ and $y^{+}$belongs to the linearity interval just to the right of $y$. Hence, the theorem follows.

The corresponding results for $g$ are stated below.

Theorem 17 Let $\bar{\mu}$ be arbitrary and let $x^{*}$ be any optimal solution of $\left(P_{\bar{\mu}}\right)$. Then the extreme points of the linearity interval $\left[\mu_{1}, \mu_{2}\right]$ containing $\bar{\mu}$ follow from

$$
\begin{aligned}
& \mu_{1}=\min _{\mu, y, s}\left\{\mu: A^{T} y+s=c+\mu \Delta c, s \geq 0, s^{T} x^{*}=0\right\} \\
& \mu_{2}=\max _{\mu, y, s}\left\{\mu: A^{T} y+s=c+\mu \Delta c, s \geq 0, s^{T} x^{*}=0\right\} .
\end{aligned}
$$

Theorem 18 Let $\bar{\mu}$ be a transition-point and let $x^{*}$ be a strictly complementary optimal solution of $\left(P_{\bar{\mu}}\right)$. Then the numbers $\mu_{1}$ and $\mu_{2}$ given by Theorem 17 satisfies $\mu_{1}=\mu_{2}=\bar{\mu}$. 


\subsection{Left and right derivatives of the value function and optimal sets in a transition- point}

In this section we show that the transition-points occur exactly where the the optimal value function is not differentiable. We have already seen that the optimal set remains constant on linearity intervals. We first deal with the differentiability of $f(\lambda)$.

If the domain of $f$ has a right extreme point then we may consider the right derivative at this point to be $\infty$, and if the domain of $f$ has a left extreme point the left derivative at this point may be taken $-\infty$. Then we may say that $\lambda$ is a transition-point of $f$ if and only if the left and the right derivative of $f$ at $\lambda$ are different. This follows from the definition of a transition-point. Denoting the left and the right derivative as $f_{-}^{\prime}(\lambda)$ and $f_{+}^{\prime}(\lambda)$ respectively, the convexity of $f$ implies that at a transition-point $\lambda$ one has

$$
f_{-}^{\prime}(\lambda)<f_{+}^{\prime}(\lambda) \text {. }
$$

If $\operatorname{dom}(f)$ has a right extreme point then it is convenient to consider the open interval at the right of this point as a linearity interval where both $f$ and its derivative are $\infty$. Similarly, if $\operatorname{dom}(f)$ has a left extreme point then we may consider the open interval at the left of this point as a linearity interval where both $f$ and its derivative are $-\infty$. Obviously, these extreme linearity intervals are characterized by the fact that on the intervals the primal problem is infeasible and the dual problem unbounded. The dual problem is unbounded if and only if the set $\mathcal{D}_{\lambda}^{*}$ of optimal solutions is empty.

Theorem 19 Let $\lambda \in \operatorname{dom}(f)$ and let $x^{*}$ be any optimal solution of $\left(P_{\lambda}\right)$. Then the derivatives at $\lambda$ satisfy

$$
\begin{aligned}
& f_{-}^{\prime}(\lambda)=\min _{y, s}\left\{\Delta b^{T} y: A^{T} y+s=c, s \geq 0, s^{T} x^{*}=0\right\} \\
& f_{+}^{\prime}(\lambda)=\max _{y, s}\left\{\Delta b^{T} y: A^{T} y+s=c, s \geq 0, s^{T} x^{*}=0\right\} .
\end{aligned}
$$

Note that we could also have uses the definition of the optimal set $\mathcal{D}_{\lambda}^{*}$ in the above theorem. The above theorem reveals that $\Delta b^{T} y$ must have the same value for all $y \in \mathcal{D}_{\lambda}^{*}$ and for all $\lambda \in\left(\lambda_{1}, \lambda_{2}\right)$. So we may state

Corollary 20 Let $\lambda \in \operatorname{dom}(f)$ belong to the linearity interval $\left(\lambda_{1}, \lambda_{2}\right)$. Then one has

$$
f^{\prime}(\lambda)=\Delta b^{T} y, \quad \forall \lambda \in\left(\lambda_{1}, \lambda_{2}\right), \forall y \in \mathcal{D}_{\lambda}^{*} .
$$

By continuity we may write

$$
f(\lambda)=b^{T} \bar{y}+\lambda \Delta b^{T} \bar{y}=b(\lambda)^{T} \bar{y}, \quad \forall \lambda \in\left[\lambda_{1}, \lambda_{2}\right] .
$$

Lemma 21 Let $\lambda \in \operatorname{dom}(f)$ belong to the linearity interval $\left(\lambda_{1}, \lambda_{2}\right)$. Moreover, let $\mathcal{D}_{\left(\lambda_{1}, \lambda_{2}\right)}^{*}:=\mathcal{D}_{\lambda}^{*}$ for arbitrary $\lambda \in\left(\lambda_{1}, \lambda_{2}\right)$. Then one has

$$
\mathcal{D}_{\left(\lambda_{1}, \lambda_{2}\right)}^{*} \subseteq \mathcal{D}_{\lambda_{1}}^{*}, \quad \mathcal{D}_{\left(\lambda_{1}, \lambda_{2}\right)}^{*} \subseteq \mathcal{D}_{\lambda_{2}}^{*} .
$$

Corollary 22 Let $\lambda$ be a nonextreme transition-point of $f$ and let $\lambda^{+}$belong to the open linearity interval just to the right of $\lambda$ and $\lambda^{-}$to the open linearity interval just to the left of $\lambda$. Then we have

$$
\mathcal{D}_{\lambda^{-}}^{*} \subset \mathcal{D}_{\lambda}^{*}, \quad \mathcal{D}_{\lambda^{+}}^{*} \subset \mathcal{D}_{\lambda}^{*}, \quad \mathcal{D}_{\lambda^{-}}^{*} \cap \mathcal{D}_{\lambda^{+}}^{*}=\phi,
$$

where the inclusions are strict.

Two other almost obvious consequences of the above results are the following corollaries. 
Corollary 23 Let $\lambda$ be a nonextreme transition-point of $f$ and let $\lambda^{+}$and $\lambda^{-}$be as defined in Corollary 22. Then we have

$$
\mathcal{D}_{\lambda^{-}}^{*}=\left\{y \in \mathcal{D}_{\lambda}^{*}: \Delta b^{T} y=\Delta b^{T} y^{-}\right\}, \quad \mathcal{D}_{\lambda^{+}}^{*}=\left\{y \in \mathcal{D}_{\lambda}^{*}: \Delta b^{T} y=\Delta b^{T} y^{+}\right\} .
$$

Corollary 24 Let $\lambda$ be a nonextreme transition-point of $f$ and let $\lambda^{+}$and $\lambda^{-}$be as defined in Corollary 22. Then

$$
\operatorname{dim} \mathcal{D}_{\lambda^{-}}^{*}<\operatorname{dim} \mathcal{D}_{\lambda}^{*}, \quad \operatorname{dim} \mathcal{D}_{\lambda^{+}}^{*}<\operatorname{dim} \mathcal{D}_{\lambda^{*}}^{*}
$$

The picture becomes more complete now. Note that Theorem 19 is valid for any value of $\lambda$ in the domain of $f$. The theorem re-establishes that at a 'non-transition' point, where the left and right derivative of $f$ are equal, the value of $\Delta b^{T} y$ is constant when $y$ runs through the dual optimal set $\mathcal{D}_{\lambda}^{*}$. But it also makes clear that at a transition-point, where the two derivatives are different, $\Delta b^{T} y$ is not constant when $y$ runs through the dual optimal set $\mathcal{D}_{\lambda}^{*}$. Then the extreme values of $\Delta b^{T} y$ yield the left and the right derivative of $f$ at $\lambda$; the left derivative is the minimum and the right derivative the maximal value of $\Delta b^{T} y$ when $y$ runs through the dual optimal set $\mathcal{D}_{\lambda}^{*}$.

The reverse of Theorem 9 also holds. This is stated in the following theorem.

Theorem 25 If $f(\lambda)$ is linear on the interval $\left[\lambda_{1}, \lambda_{2}\right]$, where $\lambda_{1}<\lambda_{2}$, then the dual optimal set $\mathcal{D}_{\lambda}^{*}$ is constant for $\lambda \in\left(\lambda_{1}, \lambda_{2}\right)$.

If $\bar{\lambda}$ is not a transition-point then there is only one linearity interval containing $\bar{\lambda}$, and hence this must be the linearity interval $\left[\lambda_{1}, \lambda_{2}\right]$, as given by Theorem 15 . It may be worthwhile to point out that if $\bar{\lambda}$ is a transition-point, however, there are three linearity intervals containing $\bar{\lambda}$, namely the singleton interval $[\bar{\lambda}, \bar{\lambda}]$ and the two surrounding linearity intervals. In that case, the linearity interval $\left[\lambda_{1}, \lambda_{2}\right]$ given by Theorem 15 may be any of these three intervals, and which of the three intervals is gotten depends on the given optimal solution $\left(y^{*}, s^{*}\right)$ of $\left(D_{\bar{\lambda}}\right)$. It can easily be understood that the linearity interval at the right of $\bar{\lambda}$ is found if $\left(y^{*}, s^{*}\right)$ happens to be optimal on the right linearity interval. This occurs exactly when $\Delta b^{T} y^{*}=f_{+}^{\prime}(\bar{\lambda})$, due to Corollary 23. Similarly, the linearity interval at the left of $\bar{\lambda}$ is found if $\left(y^{*}, s^{*}\right)$ is optimal on the left linearity interval and this occurs exactly when $\Delta b^{T} y^{*}=f_{-}^{\prime}(\bar{\lambda})$, also due to Corollary 23. Finally, if

$$
f_{-}^{\prime}(\bar{\lambda})<\Delta b^{T} y^{*}<f_{+}^{\prime}(\bar{\lambda})
$$

then we have $\lambda_{1}=\lambda_{2}=\bar{\lambda}$ in Theorem 15. The last situation seems to be most informative. It clearly indicates that $\bar{\lambda}$ is a transition-point of $f$, which is not apparent in the other two situations. Knowing that $\bar{\lambda}$ is a transition-point of $f$ we can find the two one-sided derivatives of $f$ at $\bar{\lambda}$ as well as optimal solutions for the two surrounding interval at $\bar{\lambda}$ from Theorem 19.

Remark 1 It is interesting to consider the dual optimal set $\mathcal{D}_{\lambda}^{*}$ when $\lambda$ runs from $-\infty$ to $\infty$. Left from the smallest transition-point (the transition-point for which $\lambda$ is minimal) the set $\mathcal{D}_{\lambda}^{*}$ is constant. It may happen that $\mathcal{D}_{\lambda}^{*}$ is empty there, due to the absence of optimal solutions for these small values of $\lambda$. This occurs if $\left(D_{\lambda}\right)$ is unbounded (which means that $\left(P_{\lambda}\right)$ is infeasible) for the values of $\lambda$ on the most left open linearity interval. Then, at the first transition-point, the set $\mathcal{D}_{\lambda}^{*}$ increases to a larger set, and when passing to the next open linearity interval the set $\mathcal{D}_{\lambda}^{*}$ becomes equal to a proper subset of this enlarged set. This process repeats itself at every new transitionpoint: at a transition-point of $f$ the dual optimal set expends itself and when passing to the next open linearity interval it shrinks to a proper subset of the enlarged set. Since the derivative of $f$ is monotonically increasing when $\lambda$ runs from $-\infty$ to $\infty$ every new dual optimal set arising in this way differs from all previous ones. In other words, every transition-point of $f$ and every linearity interval of $f$ has its own dual optimal set. 
Each of the above results has a dual analogy for $g(\mu)$.

Lemma 26 Let $\mu$ belong to the interior of $\operatorname{dom}(g)$ and let $\mu^{+}$belong to the open linearity interval just to the right of $\mu$ and $\mu^{-}$to the open linearity interval just to the left of $\mu$. Moreover, let $x^{+} \in \mathcal{P}_{\mu^{+}}^{*}$ and $x^{-} \in \mathcal{P}_{\mu^{-}}^{*}$. Then one has

$$
\begin{aligned}
& g_{-}^{\prime}(\mu)=\max _{x}\left\{\Delta c^{T} x: x \in \mathcal{P}_{\mu}^{*}\right\}=\Delta c^{T} x^{-} \\
& g_{+}^{\prime}(\mu)=\min _{x}\left\{\Delta c^{T} x: x \in \mathcal{P}_{\mu}^{*}\right\}=\Delta c^{T} x^{+} .
\end{aligned}
$$

Theorem 27 Let $\mu \in \operatorname{dom}(g)$ and let $\left(y^{*}, s^{*}\right)$ be any optimal solution of $\left(D_{\mu}\right)$. Then the derivatives at $\mu$ satisfy

$$
\begin{aligned}
& g_{-}^{\prime}(\mu)=\max _{x}\left\{\Delta c^{T} x: A x=b, x \geq 0, x^{T} s^{*}=0\right\} \\
& g_{+}^{\prime}(\mu)=\min _{x}\left\{\Delta c^{T} x: A x=b, x \geq 0, x^{T} s^{*}=0\right\} .
\end{aligned}
$$

Corollary 28 Under the hypothesis of Theorem 25 one has

$$
g^{\prime}(\mu)=\Delta c^{T} x, \quad \forall \mu \in\left(\mu_{1}, \mu_{2}\right), \forall x \in \mathcal{P}_{\mu}^{*}
$$

Corollary 29 Let $\mu \in \operatorname{dom}(g)$ belong to the linearity interval $\left(\mu_{1}, \mu_{2}\right)$. Moreover, let $\mathcal{P}_{\left(\mu_{1}, \mu_{2}\right)}^{*}:=\mathcal{P}_{\mu}^{*}$ for arbitrary $\mu \in\left(\mu_{1}, \mu_{2}\right)$. Then one has

$$
\mathcal{P}_{\left(\mu_{1}, \mu_{2}\right)}^{*} \subseteq \mathcal{P}_{\mu_{1}}^{*}, \quad \mathcal{P}_{\left(\mu_{1}, \mu_{2}\right)}^{*} \subseteq \mathcal{P}_{\mu_{2}}^{*}
$$

Corollary 30 Let $\mu$ be a nonextreme transition-point of $g$ and let $\mu^{+}$belong to the open linearity interval just to the right of $\mu$ and $\mu^{-}$to the open linearity interval just to the left of $\mu$. Then we have

$$
\mathcal{P}_{\mu^{-}}^{*} \subset \mathcal{P}_{\mu}^{*}, \quad \mathcal{P}_{\mu^{+}}^{*} \subset \mathcal{P}_{\mu}^{*}, \quad \mathcal{P}_{\mu^{-}}^{*} \cap \mathcal{P}_{\mu^{+}}^{*}=\phi
$$

where the inclusions are strict.

Corollary 31 Let $\mu$ be a nonextreme transition-point of $g$ and let $\mu^{+}$and $\mu^{-}$be as defined in Corollary 30. Then we have

$$
\begin{aligned}
& \mathcal{P}_{\mu^{-}}^{*}=\left\{x \in \mathcal{P}_{\mu}^{*}: \Delta c^{T} x=\Delta b^{T} x^{-}\right\}, \\
& \mathcal{P}_{\mu^{+}}^{*}=\left\{x \in \mathcal{P}_{\mu}^{*}: \Delta c^{T} x=\Delta b^{T} x^{+}\right\} .
\end{aligned}
$$

Corollary 32 Let $\mu$ be a nonextreme transition-point of $g$ and let $\mu^{+}$and $\mu^{-}$be as defined in Corollary 30 . Then

$$
\operatorname{dim} \mathcal{P}_{\mu^{-}}^{*}<\operatorname{dim} \mathcal{P}_{\mu}^{*}, \quad \operatorname{dim} \mathcal{P}_{\mu^{+}}^{*}<\operatorname{dim} \mathcal{P}_{\mu}^{*}
$$

Theorem 33 If $g(\mu)$ is linear on the interval $\left[\mu_{1}, \mu_{2}\right]$, where $\mu_{1}<\mu_{2}$, then the primal optimal set $\mathcal{P}_{\mu}^{*}$ is constant for $\mu \in\left(\mu_{1}, \mu_{2}\right)$.

\subsection{Computing the optimal value function}

Using the results of the previous sections, we present in this section an algorithm which yields the optimal value function for a one-dimensional perturbation of the vector $b$ or the vector $c$. We first deal with a one-dimensional perturbation of the vector $b$ with a scalar multiple of the vector $\Delta b$; we state the algorithm for the calculation of the optimal value function and that the algorithm finds all the transition-points and linearity intervals of it. Having done this it is clear how to treat 
a one-dimensional perturbation of the vector $c$; we also state the corresponding algorithm and its convergence results.

Assume that we have given optimal solutions $x^{*}$ of $(P)$ and $\left(y^{*}, s^{*}\right)$ of $(D)$. Using the notation of the previous sections, the problems $\left(P_{\lambda}\right)$ and its dual $\left(D_{\lambda}\right)$ arise by replacing the vector $b$ by $b(\lambda)=$ $b+\lambda \Delta b$; the optimal value of these problems is denoted as $f(\lambda)$. So we have $f(0)=c^{T} x^{*}=b^{T} y^{*}$. The domain of the optimal value function is $(-\infty, \infty)$ and $f(\lambda)=\infty$ if and only if $\left(D_{\lambda}\right)$ is unbounded. Recall from Theorem 11 that $f(\lambda)$ is convex and piecewise linear. Algorithm 1 determines $f$ on the nonnegative part of the real line. We leave it to the reader to find some straightforward modifications of the algorithm yielding an algorithm which generates $f$ on the other part of the real line.

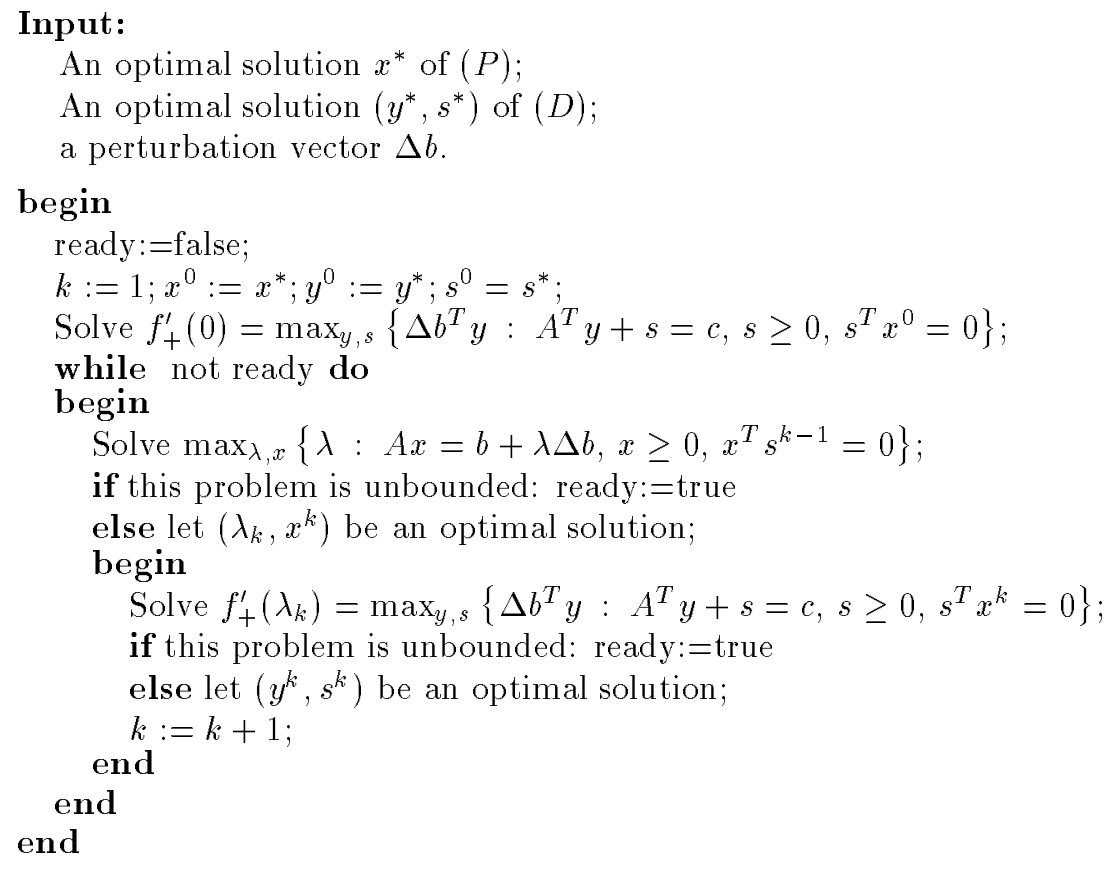

Algorithm 1: Optimal Value Function $f(\lambda), \lambda \geq 0$

The following theorem states that Algorithm 1 finds the successive transition-points of $f$ on the nonnegative part of the real line, as well as the slopes of $f$ on the successive linearity intervals.

Theorem 34 Algorithm 1 terminates after a finite number of iterations. If $K$ is the number of iterations upon termination, then $\lambda_{1}, \lambda_{2}, \cdots, \lambda_{K}$ are the successive transition-points of $f$ on the nonnegative real line. The optimal value at $\lambda_{k}(1 \leq k \leq K)$ is given by $c^{T} x^{k}$, and the slope of $f$ on the interval $\left(\lambda_{k}, \lambda_{k+1}\right)(1 \leq k<K)$ by $\Delta b^{T} y^{k}$.

From Algorithm 1 we find the linearity intervals and the slope of $f$ on these intervals. The optimal value function can now easily be drawn with these ingredients.

When perturbing the vector $c$ with a scalar multiple of $\Delta c$ to $c(\mu)=c+\mu \Delta c$ the algorithm for the calculation of the optimal value function $g(\mu)$ can be stated as in Algorithm 2 (recall that $g$ is concave). Algorithm 2 finds the successive transition-points of $g$ on the nonnegative real line as well as the slopes of $g$ on the successive linearity intervals. 


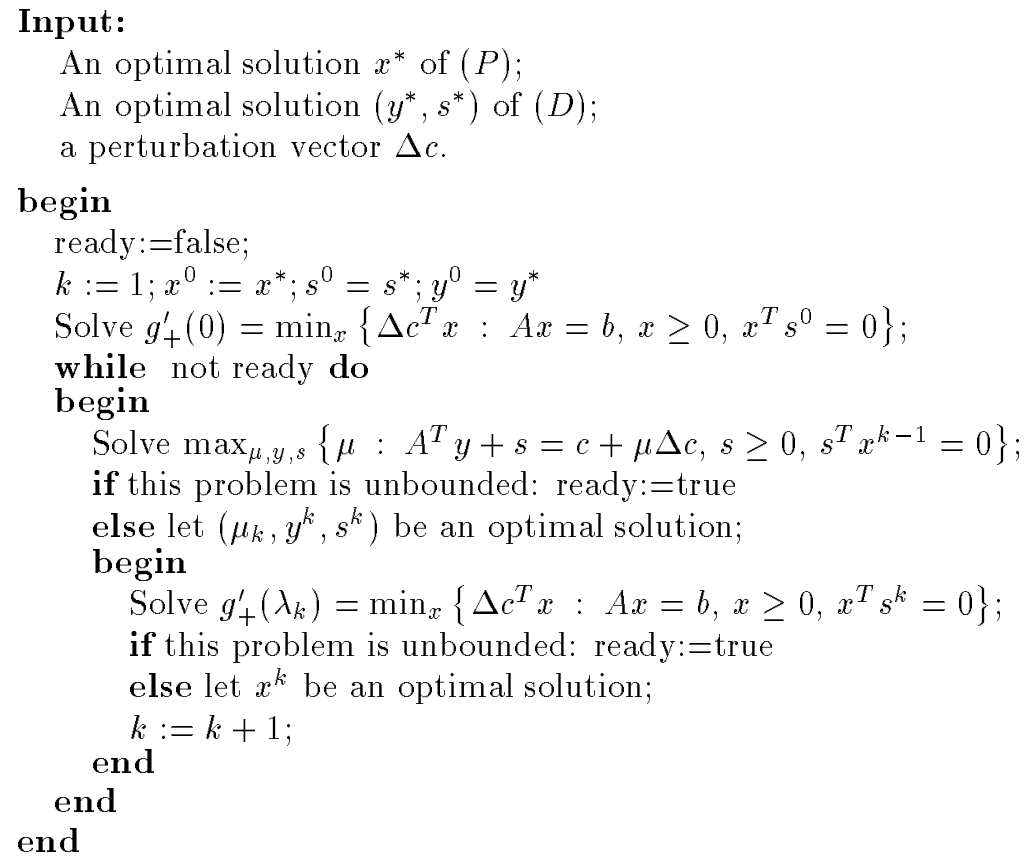

Algorithm 2: Optimal Value Function $g(\mu), \mu \geq 0$

Theorem 35 Algorithm 2 terminates after a finite number of iterations. If $K$ is the number of iterations upon termination, then $\mu_{1}, \mu_{2}, \cdots, \mu_{K}$ are the successive transition-points of $g$ on the nonnegative real line. The optimal value at $\mu_{k}(1 \leq k \leq K)$ is given by $b^{T} y^{k}$, and the slope of $g$ on the interval $\left(\mu_{k}, \mu_{k+1}\right)(1 \leq k<K)$ by $\Delta c^{T} x^{k}$.

\section{$5 \quad$ Parametric Quadratic Programming}

In this section we start to investigate the effect of changes in $b$ and $c$ on the optimal value $z(b, c)$ of $(Q P)$ and $(Q D)$. Again, as in LP, we consider one-dimensional parametric perturbations of $b$ and $c$. So we want to study

$$
z(b+\lambda \Delta b, c+\mu \Delta c)
$$

as a function of the parameters $\lambda$ and $\mu$, where $\Delta b$ and $\Delta c$ are given perturbation vectors. So, again the vectors $b$ and $c$ are fixed, and the variations come from the parameters $\lambda$ and $\mu$. The perturbed problems are denoted as $\left(\mathrm{QP}_{\lambda}\right)$ and $\left(\mathrm{QD}_{\mu}\right)$, and their feasible regions as $\mathcal{Q P} \mathcal{P}_{\lambda}$ and $\mathcal{Q D} \mathcal{D}_{\mu}$ respectively. The dual problem of $\left(\mathrm{QP}_{\lambda}\right)$ is denoted as $\left(\mathrm{QD}_{\lambda}\right)$ and the dual problem of $\left(\mathrm{QD}_{\mu}\right)$ is denoted as $\left(\mathrm{QP}_{\mu}\right)$. Observe that the feasible region of $\left(\mathrm{QD}_{\lambda}\right)$ is simply $\mathcal{Q D}$ and the feasible region of $\left(\mathrm{QP}_{\mu}\right)$ is simply $\mathcal{Q P}$. Again, we use the superscript * to refer to the optimal set of each of these problems.

We assume that $b$ and $c$ are such that $(Q P)$ and $(Q D)$ are both feasible. Then, $z(b, c)$ is again well defined and finite. We use the following notations again:

$$
\begin{gathered}
b(\lambda):=b+\lambda \Delta b, \quad c(\mu):=c+\mu \Delta c, \\
f(\lambda):=z(b(\lambda), c), \quad g(\mu):=z(b, c(\mu)) .
\end{gathered}
$$

The domain of the parameters $\lambda$ and $\mu$ is again taken as large as possible. Let us consider the domain of $f$. This function is defined as long as $z(b(\lambda), c)$ is well defined. Therefore, $f(\lambda)$ is well defined if 
the dual problem $\left(\mathrm{QD}_{\lambda}\right)$ has an optimal solution and $f(\lambda)$ is not defined (or equals infinity) if the dual problem $\left(\mathrm{QD}_{\lambda}\right)$ is unbounded. Using the Duality Theorem it follows that $f(\lambda)$ is well defined if and only if the primal problem $\left(\mathrm{QP}_{\lambda}\right)$ is feasible. In exactly the same way it can be understood that the domain of $g$ consists of all $\mu$ for which $\left(Q D_{\mu}\right)$ is feasible (hence $\left(Q P_{\mu}\right)$ bounded). Note the new meaning of the functions $f$ and $g$. We prefer to use the same notation as in the preceding section. However, these functions are defined differently.

Lemma 36 The domains of $f$ and $g$ are convex.

Proof: We give the proof for $f$. The proof for $g$ is similar and therefore omitted. Let $\lambda_{1}, \lambda_{2} \in$ $\operatorname{dom}(f)$ and $\lambda_{1}<\lambda<\lambda_{2}$. Then $f\left(\lambda_{1}\right)$ and $f\left(\lambda_{2}\right)$ are finite, which means that both $\mathcal{Q P} \lambda_{\lambda_{1}}$ and $\mathcal{Q P} \mathcal{P}_{\lambda_{2}}$ are nonempty. Let $x^{1} \in \mathcal{Q P} \mathcal{P}_{\lambda_{1}}$ and $x^{2} \in \mathcal{Q P} \mathcal{P}_{\lambda_{2}}$. Then $x^{1}$ and $x^{2}$ are nonnegative and

$$
A x^{1}=b+\lambda_{1} \Delta b, \quad A x^{2}=b+\lambda_{2} \Delta b .
$$

Now consider

$$
x:=x^{1}+\frac{\lambda-\lambda_{1}}{\lambda_{2}-\lambda_{1}}\left(x^{2}-x^{1}\right)=\frac{\left(\lambda_{2}-\lambda\right) x^{1}+\left(\lambda-\lambda_{1}\right) x^{2}}{\lambda_{2}-\lambda_{1}} .
$$

Note that $x$ is a convex combination of $x^{1}$ and $x^{2}$ and hence $x$ is nonnegative. We proceed by showing that $x \in \mathcal{Q P} \mathcal{P}_{\lambda}$. Using that $A\left(x^{2}-x^{1}\right)=\left(\lambda_{2}-\lambda_{1}\right) \Delta b$ this goes as follows:

$$
\begin{aligned}
A x & =A x^{1}+\frac{\lambda-\lambda_{1}}{\lambda_{2}-\lambda_{1}} A\left(x^{2}-x^{1}\right) \\
& =b+\lambda_{1} \Delta b+\frac{\lambda-\lambda_{1}}{\lambda_{2}-\lambda_{1}}\left(\lambda_{2}-\lambda_{1}\right) \Delta b \\
& =b+\lambda_{1} \Delta b+\left(\lambda-\lambda_{1}\right) \Delta b \\
& =b+\lambda \Delta b .
\end{aligned}
$$

This proves that $\left(\mathrm{QP}_{\lambda}\right)$ is feasible and hence $\lambda \in \operatorname{dom}(f)$, completing the proof.

Lemma 37 The functions $f$ and $g$ are convex.

Proof: We present the proof only for $f$, the proof for $g$ is similar. Let $\lambda_{1}, \lambda_{2}$ be elements of the interior of the domain of $f$. Let $\alpha \in(0,1)$ be given and define $\lambda_{\alpha}:=\alpha \lambda_{1}+(1-\alpha) \lambda_{2}$. Then we have

$$
\begin{aligned}
f\left(\lambda_{\alpha}\right)= & \alpha\left(\left(b+\lambda_{1} \Delta b\right)^{T} y^{\left(\lambda_{\alpha}\right)}-\frac{1}{2}\left(x^{\left(\lambda_{\alpha}\right)}\right)^{T} Q x^{\left(\lambda_{\alpha}\right)}\right)+ \\
& (1-\alpha)\left(\left(b+\lambda_{2} \Delta b\right)^{T} y^{\left(\lambda_{\alpha}\right)}-\frac{1}{2}\left(x^{\left(\lambda_{\alpha}\right)}\right)^{T} Q x^{\left(\lambda_{\alpha}\right)}\right) \\
\leq & \alpha f\left(\lambda_{1}\right)+(1-\alpha) f\left(\lambda_{2}\right),
\end{aligned}
$$

where the inequality holds since the feasible set of $\left(\mathrm{QD}_{\lambda}\right)$ is independent of $\lambda$.

Lemma 38 The complements of the domains of $f$ and $g$ are open intervals of the real line. 
Proof: Let $\lambda$ belong to the complement of the domain of $f$. This means that $\left(\mathrm{QD}_{\lambda}\right)$ is unbounded. This is equivalent to the existence of a vector $z$, such that

$$
A^{T} z \leq 0,(b+\lambda \Delta b)^{T} z>0 .
$$

Fixing $z$ and considering $\lambda$ as a variable, the set of all $\lambda$ satisfying $(b+\lambda \Delta b)^{T} z>0$ is an open interval. For all $\lambda$ in this interval $\left(\mathrm{QD}_{\lambda}\right)$ is unbounded. Hence, the complement of the domain of $f$ is open. This concludes the proof.

A consequence of the last two lemmas is the next theorem (cf. Theorem 8) which requires no further proof.

Theorem 39 The domains of $f$ and $g$ are closed intervals on the real line.

\subsection{Optimal value function and optimal partitions on a curvy-linearity interval}

In this section we show that the optimal value functions $f(\lambda)$ and $g(\mu)$ are piecewise quadratic on their domains. The pieces correspond to intervals where the partition is constant. In LP these results are given in terms of the optimal primal and dual sets. In QP this is not possible since these sets are intertwined. Neither $\left(\mathrm{QP}_{\mu}\right)$ nor $\left(\mathrm{QD}_{\lambda}\right)$ is constant when $\lambda$ or $\mu$ vary. The proofs for LP are obtained by using the characterization of the optimal set by the optimal partition (see Section 6.3). For any $\lambda$ in the domain of $f$ we denote the optimal set of $\left(\mathrm{QP}_{\lambda}\right)$ as $\mathcal{Q P}_{\lambda}^{*}$ and the optimal set of $\left(\mathrm{QD}_{\lambda}\right)$ as $\mathcal{Q} \mathcal{D}_{\lambda}^{*}$. The first theorem (cf. Theorem 9) shows that the partition is constant on certain intervals and that $f$ is quadratic on these intervals.

Theorem 40 Let $\lambda_{1}$ and $\lambda_{2}>\lambda_{1}$ be such that $\pi_{\lambda_{1}}=\pi_{\lambda_{2}}$. Then $\pi_{\lambda}$ is constant for all $\lambda \in\left[\lambda_{1}, \lambda_{2}\right]$ and $f(\lambda)$ is quadratic on the interval $\left[\lambda_{1}, \lambda_{2}\right]$.

Proof: Without loss of generality we assume that $\lambda_{1}=0$ and $\lambda_{2}=1$. Let $\left(x^{\left(\lambda_{1}\right)},\left(u^{\left(\lambda_{1}\right)}, y^{\left(\lambda_{1}\right)}, s^{\left(\lambda_{1}\right)}\right)\right)$ and $\left(x^{\left(\lambda_{2}\right)},\left(u^{\left(\lambda_{2}\right)}, y^{\left(\lambda_{2}\right)}, s^{\left(\lambda_{2}\right)}\right)\right)$ be maximal complementary solutions for the respective problems. We assume that $u^{\left(\lambda_{1}\right)}=x^{\left(\lambda_{1}\right)}$ and $u^{\left(\lambda_{2}\right)}=x^{\left(\lambda_{2}\right)}$ and define for $\lambda \in(0,1)$

$$
\begin{aligned}
x(\lambda) & :=(1-\lambda) x^{\left(\lambda_{1}\right)}+\lambda x^{\left(\lambda_{2}\right)}, \\
y(\lambda) & :=(1-\lambda) y^{\left(\lambda_{1}\right)}+\lambda y^{\left(\lambda_{2}\right)}, \\
s(\lambda) & :=(1-\lambda) s^{\left(\lambda_{1}\right)}+\lambda s^{\left(\lambda_{2}\right)} .
\end{aligned}
$$

It is easy to see that $A x(\lambda)=b+\lambda \Delta b$ and $x(\lambda) \geq 0$. Also

$$
A^{T} y(\lambda)+s(\lambda)-Q x(\lambda)=(1-\lambda) c+\lambda c=c,
$$

and $s(\lambda) \geq 0$. So $(x(\lambda), y(\lambda), s(\lambda))$ is feasible for $\left(\mathrm{QP}_{\lambda}\right)$ and $\left(\mathrm{QD}_{\lambda}\right)$. Since $\pi_{0}=\pi_{1}$, we have $x(\lambda)^{T} s(\lambda)=0$, hence the proposed solution is optimal for $\left(\mathrm{QP}_{\lambda}\right)$ and $\left(\mathrm{QD}_{\lambda}\right)$. Using the support of $x(\lambda)$ and $s(\lambda), B$ and $N$ respectively, and their complement $T$, this implies

$$
B \subseteq B_{\lambda}, N \subseteq N_{\lambda} \text { and } T \supseteq T_{\lambda} .
$$

We now show that equality holds. Assuming to the contrary that $T \supset T_{\lambda}$, there exists a maximal complementary solution $\left(x^{(\lambda)}, y^{(\lambda)}, s^{(\lambda)}\right)$ of $\left(\mathrm{QP}_{\lambda}\right)$ and $\left(\mathrm{QD}_{\lambda}\right)$ such that

$$
\left(x^{(\lambda)}\right)_{i}+\left(s^{(\lambda)}\right)_{i}>0 \text { for some } i \in T, i \notin T_{\lambda} .
$$

Let us now define for $\epsilon>0$

$$
\begin{gathered}
\bar{x}(\epsilon):=x^{\left(\lambda_{2}\right)}+\epsilon\left(x^{\left(\lambda_{2}\right)}-x^{\left(\lambda_{1}\right)}\right), \\
\bar{y}(\epsilon):=y^{\left(\lambda_{2}\right)}+\epsilon\left(y^{\left(\lambda_{2}\right)}-y^{\left(\lambda_{1}\right)}\right), \\
\bar{s}(\epsilon):=s^{\left(\lambda_{2}\right)}+\epsilon\left(s^{\left(\lambda_{2}\right)}-s^{\left(\lambda_{1}\right)}\right) .
\end{gathered}
$$


For some $\bar{\epsilon}>0$ small enough it holds

$$
\bar{x}(\bar{\epsilon})_{B}>0, \bar{x}(\bar{\epsilon})_{N \cup T}=0, \quad \bar{s}(\bar{\epsilon})_{N}>0, \bar{s}(\bar{\epsilon})_{B \cup T}=0,
$$

from which it follows that the proposed solutions are optimal for $\left(\mathrm{QP}_{1+\bar{\epsilon}}\right)$ and $\left(\mathrm{QD}_{1+\bar{\epsilon}}\right)$. Finally, we define

$$
\begin{aligned}
& \tilde{x}:=\frac{\bar{\epsilon}}{1-\frac{\lambda}{\epsilon}+\bar{\epsilon}} x^{(\lambda)}+\frac{1-\lambda}{1-\lambda+\bar{\epsilon}} \bar{x}(\bar{\epsilon}), \\
& \tilde{y}:=\frac{1-\lambda}{1-\frac{\lambda}{\bar{\epsilon}}+\bar{\epsilon}} y^{(\lambda)}+\frac{1-\lambda}{1-\lambda+\bar{\epsilon}} \bar{y}(\bar{\epsilon}), \\
& \tilde{s}:=\frac{1-\lambda}{1-\lambda+\bar{\epsilon}} s^{(\lambda)}+\frac{1-\lambda}{1-\lambda+\bar{\epsilon}} \bar{s}(\bar{\epsilon}),
\end{aligned}
$$

which are feasible in $\left(\mathrm{QP}_{1}\right)$ and $\left(\mathrm{QD}_{1}\right)$. Also,

$$
\tilde{x}^{T} \tilde{s}=\frac{\bar{\epsilon}}{1-\lambda+\bar{\epsilon}} \frac{1-\lambda}{1-\lambda+\bar{\epsilon}}\left(\left(x^{(\lambda)}\right)^{T} \bar{s}(\bar{\epsilon})+\bar{x}(\bar{\epsilon})^{T} s^{(\lambda)}\right) .
$$

Since (3) and (5) imply

and

$$
\left(x^{(\lambda)}\right)^{T} \bar{s}(\bar{\epsilon})=\left(x^{(\lambda)}\right)_{N}^{T} \bar{s}(\bar{\epsilon})_{N}=0
$$

$$
\left(s^{(\lambda)}\right)^{T} \bar{x}(\bar{\epsilon})=\left(s^{(\lambda)}\right)_{B}^{T} \bar{x}(\bar{\epsilon})_{B}=0,
$$

$(\tilde{x}, \tilde{y}, \tilde{s})$ is optimal for $\left(\mathrm{QP}_{1}\right)$ and $\left(\mathrm{QD}_{1}\right)$. However, if (4) would hold, we would have a solution of $\left(\mathrm{QP}_{1}\right)$ and $\left(\mathrm{QD}_{1}\right)$ with either $(\tilde{x})_{i}>0$ or $(\tilde{s})_{i}>0$ for $i \in T$, contradicting the definition of $(B, N, T)$. Thus we conclude $T_{\lambda}=T$. Using (3) the first part of the theorem follows.

The second part can now be proven almost straightforwardly. From the proof of the first part we know that $(x(\lambda), y(\lambda), s(\lambda))$ defined in (2) is optimal in $\left(\mathrm{QD}_{\lambda}\right)$ for $\lambda \in(0,1)$. Hence

$$
\begin{aligned}
f(\lambda)= & (b+\lambda \Delta b)^{T} y(\lambda)-\frac{1}{2} x(\lambda)^{T} Q x(\lambda) \\
= & f(0)+\lambda \Delta b^{T} y^{\left(\lambda_{1}\right)}+\lambda b^{T}\left(y^{\left(\lambda_{2}\right)}-y^{\left(\lambda_{1}\right)}\right)+\lambda^{2} \Delta b^{T}\left(y^{\left(\lambda_{2}\right)}-y^{\left(\lambda_{1}\right)}\right)- \\
& \lambda\left(x^{\left(\lambda_{2}\right)}-x^{\left(\lambda_{1}\right)}\right)^{T} Q x^{\left(\lambda_{1}\right)}-\frac{1}{2} \lambda^{2}\left(x^{\left(\lambda_{2}\right)}-x^{\left(\lambda_{1}\right)}\right)^{T} Q\left(x^{\left(\lambda_{2}\right)}-x^{\left(\lambda_{1}\right)}\right) .
\end{aligned}
$$

Note that

$$
\begin{aligned}
A\left(x^{\left(\lambda_{2}\right)}-x^{\left(\lambda_{1}\right)}\right) & =\Delta b \\
A^{T}\left(y^{\left(\lambda_{2}\right)}-y^{\left(\lambda_{1}\right)}\right)+s^{\left(\lambda_{2}\right)}-s^{\left(\lambda_{1}\right)} & =Q\left(x^{\left(\lambda_{2}\right)}-x^{\left(\lambda_{1}\right)}\right) .
\end{aligned}
$$

Multiplying the second equation with $x^{\left(\lambda_{2}\right)}-x^{\left(\lambda_{1}\right)}$ respectively with $x^{\left(\lambda_{1}\right)}$, and using the first, gives

$$
\begin{aligned}
\Delta b^{T}\left(y^{\left(\lambda_{2}\right)}-y^{\left(\lambda_{1}\right)}\right) & =\left(x^{\left(\lambda_{2}\right)}-x^{\left(\lambda_{1}\right)}\right)^{T} Q\left(x^{\left(\lambda_{2}\right)}-x^{\left(\lambda_{1}\right)}\right) \\
b^{T}\left(y^{\left(\lambda_{2}\right)}-y^{\left(\lambda_{1}\right)}\right) & =\left(x^{\left(\lambda_{1}\right)}\right)^{T} Q\left(x^{\left(\lambda_{2}\right)}-x^{\left(\lambda_{1}\right)}\right) .
\end{aligned}
$$

So we may write $f(\lambda)$ as

$$
f(\lambda)=f(0)+\lambda \Delta b^{T} y^{\left(\lambda_{1}\right)}+\frac{1}{2} \lambda^{2} \Delta b^{T}\left(y^{\left(\lambda_{2}\right)}-y^{\left(\lambda_{1}\right)}\right) .
$$

This concludes the proof.

The function $f$ on $\left[\lambda_{1}, \lambda_{2}\right]$ is explicitly given by the following formula

$$
\begin{gathered}
f(\lambda)=\frac{\lambda_{2}}{\Delta \lambda}\left(b^{T} y^{\left(\lambda_{1}\right)}\right)-\frac{1}{2} \frac{\lambda_{2}}{\Delta \lambda}\left(x^{\left(\lambda_{1}\right)}\right)^{T} Q x^{\left(\lambda_{1}\right)}+\frac{\lambda_{1}}{\Delta \lambda}\left(b^{T} y^{\lambda_{2}}\right)-\frac{1}{2} \frac{\lambda_{1}}{\Delta \lambda}\left(x^{\left(\lambda_{2}\right)}\right)^{T} Q x^{\left(\lambda_{2}\right)}+ \\
\frac{\lambda}{\Delta \lambda}\left(c^{T}\left(x^{\left(\lambda_{2}\right)}-x^{\left(\lambda_{1}\right)}\right)+\left(2-\lambda_{1}-\lambda_{2}\right) b^{T}\left(y^{\left(\lambda_{2}\right)}-y^{\left(\lambda_{1}\right)}\right)+\frac{2 \lambda_{2} \lambda_{1}}{\Delta \lambda} \Delta b^{T}\left(y^{\left(\lambda_{2}\right)}-y^{\left(\lambda_{1}\right)}\right)\right) \\
+\frac{1}{2} \frac{\lambda^{2}}{\Delta \lambda} \Delta b^{T}\left(y^{\left(\lambda_{2}\right)}-y^{\left(\lambda_{1}\right)}\right) .
\end{gathered}
$$

Note that we can now calculate the optimal value function between two subsequent transition-points.

Theorem 40 implies the following corollary. 
Corollary 41 If $\pi_{\lambda_{1}}=\pi_{\lambda_{2}}=\pi$ then $f(\lambda)$ is linear on $\left[\lambda_{1}, \lambda_{2}\right]$ if and only if $Q x^{\left(\lambda_{1}\right)}=Q x^{\left(\lambda_{2}\right)}$.

Proof: Assuming again $\lambda_{1}=0$ and $\lambda_{2}=1$, we have from the proof of Theorem 40 that $f(\lambda)$ is linear on [0,1] if and only if $\Delta b^{T}\left(y^{\left(\lambda_{2}\right)}-y^{\left(\lambda_{1}\right)}\right)=0$. Using (6) this is equivalent to

$$
\left(x^{\left(\lambda_{2}\right)}-x^{\left(\lambda_{1}\right)}\right)^{T} Q\left(x^{\left(\lambda_{2}\right)}-x^{\left(\lambda_{1}\right)}\right)=0
$$

which holds if and only if $Q\left(x^{\left(\lambda_{2}\right)}-x^{\left(\lambda_{1}\right)}\right)$.

As a consequence of Theorem 40 we have the following results (cf. Theorem 9 and Theorem 11.

Theorem 42 The domain of $f$ can be partitioned in a finite set of subintervals such that the optimal partition is constant on a subinterval.

Proof: Since the number of possible partitions is finite and the number of elements in the domain of $f$ is infinite, it follows from Theorem 40 that the domain of $f$ can be partitioned into (open) subintervals on which the partition is constant, while it is different in the singletons in between the subintervals. This implies the result.

Theorem 43 The optimal value function $f(\lambda)$ is continuous, convex and piecewise quadratic.

Proof: Corollary 41 implies that on each subinterval defined by a partition the function $f(\lambda)$ is quadratic. Since $f(\lambda)$ is convex (Lemma 37) it is continuous on the interior of its domain. It remains to be shown that the optimal value function is right-continuous and left-continuous in the left and right endpoints of the domain of $f$ respectively. To this end we consider the left endpoint of the domain of $f$ (for the right endpoint the proof is similar). Let $\lambda^{*}$ denote the left endpoint. Now, we need to proof that

$$
\lim _{\lambda \downarrow \lambda^{*}} f(\lambda)=f\left(\lambda^{*}\right)
$$

Let $(\bar{x}, \bar{y}, \bar{s})$ denote the optimal solution at the left endpoint $\lambda^{*}$. Furthermore consider the limit of $(x(\lambda), y(\lambda), s(\lambda))$ for $\lambda \downarrow \lambda^{*}$. Since the dual feasible set ( $\left.\mathrm{QD}_{\lambda}\right)$ is closed and is independent of $\lambda$, the limit point $\tilde{y}, \tilde{s}$ of $y(\lambda), s(\lambda)$ is dual feasible. Further the limit point $\tilde{x}$ of the sequence $x(\lambda)$ is feasible for $\left(\mathrm{QP}_{\lambda^{*}}\right)$. Since the sequences are complementary, the limit points are also complementary, hence optimal. Applying Lemma 4 completes the proof.

The values of $\lambda$ where the partition of the optimal value function $f(\lambda)$ changes are called transitionpoints of $f$, and any interval between two successive transition-points of $f$ is called a curvy-linearity interval of $f$. In a similar way we define transition-points and curvy-linearity intervals for $g$. Each of the above results on $f(\lambda)$ has its analogue for $g(\mu)$. We state these results without further proof. The omitted proofs are straightforward modifications of the above proofs.

Theorem 44 Let $\mu_{1}$ and $\mu_{2}>\mu_{1}$ be such that $\pi_{\mu_{1}}=\pi_{\mu_{2}}$. Then $\pi_{\mu}$ is constant for all $\mu \in\left[\mu_{1}, \mu_{2}\right]$ and $g(\mu)$ is quadratic on the interval $\left[\mu_{1}, \mu_{2}\right]$.

Corollary 45 If $\pi_{\mu_{1}}=\pi_{\mu_{2}}=\pi$ then $g(\mu)$ is linear on $\left[\lambda_{1}, \lambda_{2}\right]$ if and only if $Q x^{\left(\lambda_{1}\right)}=Q x^{\left(\lambda_{2}\right)}$.

Theorem 46 The domain of $g$ can be partitioned in a finite set of subintervals such that the optimal partition is constant on a subinterval. 


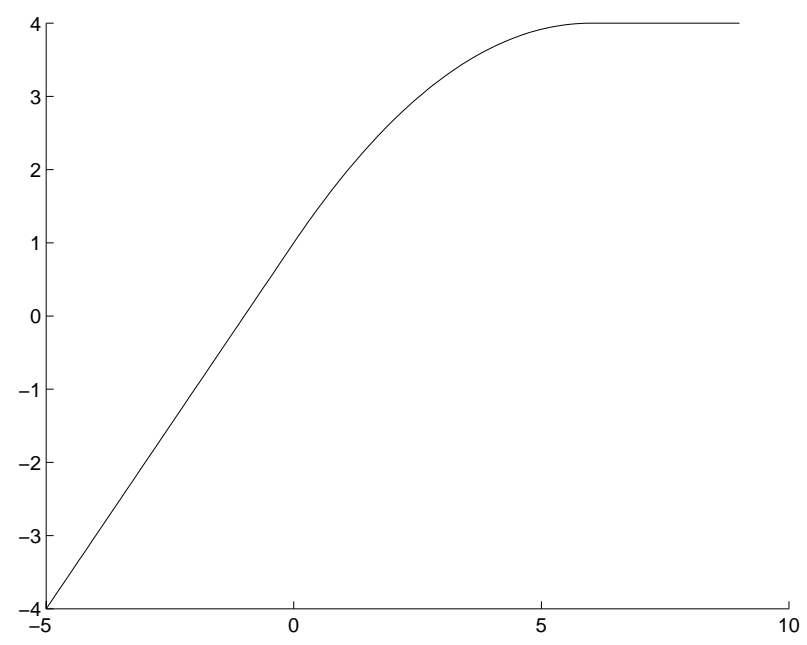

Fig. 1: Continuously differentiable value function

Theorem 47 The optimal value function $g(\mu)$ is continuous, concave and piecewise quadratic.

For parametric LP, the optimal value functions $f(\lambda)$ and $g(\mu)$ are piecewise linear and continuous (see Theorems 11 and Theorem 14). The linearity intervals are the intervals on which the optimal value function is linear and the solution sets are faces of the feasible set. The transition-points exactly correspond to the transition-points of the piecewise linear optimal value function. Thus, the optimal set changes exactly where the optimal value function is not differentiable. In QP, unlike LP, the next example shows that although the optimal partition changes in certain points, the valuefunction may be continuous differentiable in those points. Later on we show that a transition-point always corresponds to discontinuity or nonexistence of the second order derivative.

Example 2 Consider the following parametric QP model,

$$
Q=\left[\begin{array}{ll}
2 & 0 \\
0 & 1
\end{array}\right], A=\left[\begin{array}{ll}
2 & 1
\end{array}\right], c=\left[\begin{array}{l}
0 \\
1
\end{array}\right], \Delta c=\left[\begin{array}{l}
1 \\
0
\end{array}\right], b=2,
$$

\begin{tabular}{c|cccc} 
& $B$ & $N$ & $T$ & $g(\mu)$ \\
\hline$\mu<0$ & $\{1\}$ & $\{2\}$ & $\phi$ & $1+\mu$ \\
$0<\mu<6$ & $\{1,2\}$ & $\phi$ & $\phi$ & $1+\mu-\frac{1}{12} \mu^{2}$ \\
$\mu>6$ & $\{2\}$ & $\{1\}$ & $\phi$ & 4
\end{tabular}

Although the partitions in the transition-points are not given, it should be noted that these are different than in the neighbouring curvy-linearity intervals. The reader can verify that the optimal value function is continuously differentiable. In Figure 1 the optimal value function is drawn.

\subsection{Extreme points of a curvy-linearity interval}

In this section we assume that $\bar{\lambda}$ belongs to the interior of a curvy-linearity interval $\left[\lambda_{1}, \lambda_{2}\right]$. Given an optimal solution of $\left(Q D_{\bar{\lambda}}\right)$ we show how the extreme points $\lambda_{1}$ and $\lambda_{2}$ of the curvy-linearity interval containing $\bar{\lambda}$ can be found by solving two auxiliary linear optimization problems (cf. Theorem 15 ). 
Theorem 48 Let $\bar{\lambda}$ be arbitrary. Moreover, let $\left(x^{*}, y^{*}, s^{*}\right)$ be any optimal solution of $\left(\mathrm{QD}_{\bar{\lambda}}\right)$ and let $x^{*}$ be any optimal solution of $\left(\mathrm{QP}_{\bar{\lambda}}\right)$. Furthermore, define $T:=\{1, \ldots, n\} \backslash\left(\sigma\left(x^{*}\right) \cup \sigma\left(s^{*}\right)\right.$. Then the extreme points of the curvy-linearity interval $\left[\lambda_{1}, \lambda_{2}\right]$ containing $\vec{\lambda}$ follow from

$$
\begin{aligned}
& \lambda_{1}=\min _{\lambda, x, y, s}\left\{\lambda \quad: \quad \begin{array}{l}
A x=b+\lambda \Delta b, x \geq 0, x^{T} s^{*}=0, x_{T}=0 \\
\\
A^{T} y+s-Q x=c, s \geq 0, s^{T} x^{*}=0, s_{T}=0
\end{array}\right\} \\
& \lambda_{2}=\max _{\lambda, x, y, s}\left\{\lambda \quad: \quad \begin{array}{l}
A x=b+\lambda \Delta b, x \geq 0, x^{T} s^{*}=0, x_{T}=0 \\
A^{T} y+s-Q x=c, x \geq 0, s^{T} x^{*}=0, s_{T}=0
\end{array}\right.
\end{aligned}
$$

Proof: We only give the proof for $\lambda_{1}$. The proof for $\lambda_{2}$ goes in the same way and is therefore omitted. We first show that (8) is feasible. Since $\left(x^{*}, y^{*}, s^{*}\right)$ is a primal-dual optimal solution we can easily see that (8) is feasible for this solution with $\lambda=\bar{\lambda}$.

We proceed by considering the case where (8) is unbounded. Then for any $\lambda \leq \bar{\lambda}$ there exists a vector $(x, y, s)$ which satisfies $A x=b+\lambda \Delta b, x \geq 0, x^{T} s^{*}=0, x_{T}=0$ and $A^{T} y+s-Q x=c, s \geq$ $0, s^{T} x^{*}=0, s_{T}=0$. Now $(x, y, s)$ is feasible for $\left(\mathrm{QD}_{\lambda}\right)$ and $x$ is feasible for $\left(\mathrm{QP}_{\lambda}\right)$. Since $x^{T} s=0$, $x$ is optimal for $\left(\mathrm{QP}_{\lambda}\right)$ and $(x, y, s)$ is optimal for $\left(\mathrm{QD}_{\lambda}\right)$. The optimal value of both problems is given by $b(\lambda)^{T} y^{*}-\frac{1}{2}\left(u^{*}\right)^{T} Q u^{*}=b^{T} y^{*}+\lambda \Delta b^{T} y^{*}-\frac{1}{2}\left(u^{*}\right)^{T} Q u^{*}$. This means that $\lambda$ belongs to the curvy-linearity interval containing $\bar{\lambda}$. Since this holds for any $\lambda \leq \bar{\lambda}$, the left boundary of this curvy-linearity interval is $-\infty$, as it should.

It remains to deal with the case where $(8)$ has an optimal solution, say $(\tilde{\lambda}, \tilde{x}, \tilde{y}, \tilde{s})$. We then have $A \tilde{x}=b+\tilde{\lambda} \Delta b=b(\tilde{\lambda})$, and $A^{T} \tilde{y}+\tilde{s}-Q \tilde{x}=c$, so $\tilde{x}$ is feasible for $\left(Q P_{\tilde{\lambda}}\right)$. Since $(\tilde{x}, \tilde{y}, \tilde{s})$ is feasible for $\left(Q D_{\tilde{\lambda}}\right)$ and $\tilde{x}^{T} \tilde{s}=0$ it follows that $\tilde{x}$ is optimal for $\left(Q P_{\tilde{\lambda}}\right)$ and $(\tilde{x}, \tilde{y}, \tilde{s})$ is optimal for $\left(Q D_{\tilde{\lambda}}\right)$. The optimal value of both problems is given by $b(\tilde{\lambda})^{T} \tilde{y}-\frac{1}{2}(\tilde{x})^{T} Q \tilde{x}=b^{T} \tilde{y}+\tilde{\lambda} \Delta b^{T} \tilde{y}-\frac{1}{2}(\tilde{x})^{T} Q \tilde{x}$. This means that $\tilde{\lambda}$ belongs to the curvy-linearity interval containing $\bar{\lambda}$, and thus it follows that $\tilde{\lambda} \geq \lambda_{1}$.

Since every $\lambda$ on the curvy-linearity interval containing $\bar{\lambda}$ is feasible in (8) and any $\tilde{\lambda} \leq \lambda_{1}$ gives a contradiction with the definition of the partition, the proof is concluded.

The corresponding results for $g$ are stated below.

Theorem 49 Let $\bar{\mu}$ be arbitrary and let $x^{*}$ be any optimal solution of $\left(Q P_{\bar{\mu}}\right)$ and $\left(x^{*}, y^{*}, s^{*}\right)$ be any optimal solution of $\left(Q D_{\bar{\mu}}\right)$. Furthermore, define $T:=\{1, \ldots, n\} \backslash\left(\sigma\left(x^{*}\right) \cup \sigma\left(s^{*}\right)\right.$. Then the extreme points of the curvy-linearity interval $\left[\mu_{1}, \mu_{2}\right]$ containing $\bar{\mu}$ follow from

$$
\begin{aligned}
\mu_{1}=\min _{\mu, u, y, s}\{\mu: & A^{T} y+s-Q u=c+\mu \Delta c \\
& s \geq 0, s^{T} x^{*}=0, s_{T}=0, \\
& A x=b \\
& \left.x \geq 0, x^{T} s^{*}=0, x_{T}=0\right\} \\
\mu_{2}=\max _{\mu, u, y, s}\{\mu \quad: \quad & A^{T} y+s-Q u=c+\mu \Delta c \\
& s \geq 0, s^{T} x^{*}=0, s_{T}=0, \\
& A x=b \\
& \left.x \geq 0, x^{T} s^{*}=0, x_{T}=0\right\}
\end{aligned}
$$

\subsection{Left and right derivatives of the value function and optimal partitions in a transition- point}

In this section we show that the transition-points occur exactly where the first or second order derivative of the optimal value function does not exist. Moreover, we prove that when taking maximal complementary solutions for two parameter values in a curvy-linearity interval, a convex 
combination of these solutions is a maximal complementary solution for the corresponding parameter value. We first deal with the differentiability of $f(\lambda)$ (cf. Theorem 19).

If the domain of $f$ has a right extreme point, then we may consider the right derivative at this point to be $\infty$, and if the domain of $f$ has a left extreme point, the left derivative at this point may be taken $-\infty$. Denoting the left and the right derivative as $f_{-}^{\prime}(\lambda)$ and $f_{+}^{\prime}(\lambda)$ respectively, the convexity of $f$ implies that at a transition-point $\lambda$ one has

$$
f_{-}^{\prime}(\lambda) \leq f_{+}^{\prime}(\lambda)
$$

If $\operatorname{dom}(f)$ has a right extreme point then it is convenient to consider the open interval at the right of this point as a curvy-linearity interval where both $f$ and its derivative are $\infty$. Similarly, if dom $(f)$ has a left extreme point then we may consider the open interval at the left of this point as a curvylinearity interval where both $f$ and its derivative are $-\infty$. Obviously, these extreme curvy-linearity intervals are characterized by the fact that on the intervals the primal problem is infeasible and the dual problem unbounded. The dual problem is unbounded if and only if the set $\mathcal{Q D} \mathcal{D}_{\lambda}^{*}$ of optimal solutions is empty.

Theorem 50 Let $\lambda \in \operatorname{dom}(f)$ and let $x^{*}$ be any optimal solution of $\left(\mathrm{QP}_{\lambda}\right)$. Moreover, let $\left(x^{*}, y^{*}, s^{*}\right)$ be any optimal solution of $\left(\mathrm{QD}_{\lambda}\right)$. Furthermore, define $T:=\{1, \ldots, n\} \backslash\left(\sigma\left(x^{*}\right) \cup \sigma\left(s^{*}\right)\right.$. Then, the derivatives at $\lambda$ satisfy

$$
\begin{aligned}
f_{-}^{\prime}(\lambda)=\min _{x, y, s}\left\{\Delta b^{T} y:\right. & A^{T} y+s-Q x=c \\
& s \geq 0, s^{T} x^{*}=0, s_{T}=0, \\
& A x=b+\lambda \Delta b \\
& \left.x \geq 0, x^{T} s^{*}=0, x_{T}=0\right\}, \\
f_{+}^{\prime}(\lambda)=\max _{x, y, s}\left\{\Delta b^{T} y:\right. & A^{T} y+s-Q x=c \\
& s \geq 0, s^{T} x^{*}=0, s_{T}=0, \\
& A x=b+\lambda \Delta b \\
& \left.x \geq 0, x^{T} s^{*}=0, x_{T}=0\right\} .
\end{aligned}
$$

Proof: Let $\epsilon \in \mathbb{R}$ be sufficiently small. Then for any optimal solution $(x(\lambda), y(\lambda), s(\lambda))$ of $\left(\mathrm{QD}_{\lambda}\right)$ it holds

$$
\begin{aligned}
f(\lambda+\varepsilon) & =(b+(\lambda+\varepsilon) \Delta b)^{T} y^{(\lambda+\varepsilon)}-\frac{1}{2}\left(u^{(\lambda+\varepsilon)}\right)^{T} Q u^{(\lambda+\varepsilon)} \\
& \geq(b+\lambda \Delta b)^{T} y(\lambda)-\frac{1}{2} u(\lambda)^{T} Q u(\lambda)+\varepsilon \Delta b^{T} y(\lambda) \\
& =f(\lambda)+\varepsilon \Delta b^{T} y(\lambda)
\end{aligned}
$$

so the right and left derivatives at $\lambda$ satisfy

$$
\begin{aligned}
& f_{+}^{\prime}(\lambda)=\lim _{\varepsilon \downarrow 0} \frac{f(\lambda+\varepsilon)-f(\lambda)}{\varepsilon} \geq \Delta b^{T} y(\lambda), \\
& f_{-}^{\prime}(\lambda)=\lim _{\varepsilon \uparrow 0} \frac{f(\lambda+\varepsilon)-f(\lambda)}{\varepsilon} \leq \Delta b^{T} y(\lambda) .
\end{aligned}
$$

If $f$ is differentiable at $\lambda$ then we necessarily have $f^{\prime}(\lambda)=\Delta b^{T} y(\lambda)$ for any optimal solution $y(\lambda)$ of $\left(\mathrm{QD}_{\lambda}\right)$. Otherwise, note that the objective value of each $y$ feasible in $\left(\mathrm{QD}_{\lambda}\right)$ as a function of $\lambda$ is linear with slope $\Delta b^{T} y$. Since $f$ is assumed to be not differentiable in $\lambda$ there must be different optimal solutions with different slope. We restrict to proving (13), the proof of (12) is analogous. Let us consider a maximal complementary solution $\left(u^{+}(\epsilon), y^{+}(\epsilon), s^{+}(\epsilon)\right)$ of $\left(\mathrm{QD}_{\lambda+\epsilon}\right)$. Let $\epsilon>0$ be chosen to assure that $f(\lambda)$ is differentiable in $\lambda+\epsilon$. Furthermore, let us denote $u(\lambda), y(\lambda)$ and 
$s(\lambda)$, the optimal solution used in the upper part of the proof, by $u, y$ and $s$. It easily follows that $f^{\prime}(\lambda+\epsilon)=\Delta b^{T} y^{+}(\epsilon)$. Since $f^{\prime}$ is increasing ( $f$ is convex) we conclude that

$$
\Delta b^{T} y \leq f_{-}^{\prime}(\lambda) \leq \Delta b^{T} y^{+}(\epsilon)=f^{\prime}(\lambda+\epsilon) .
$$

Taking the limit of $\epsilon \downarrow 0$, it holds

$$
\Delta b^{T} y \leq f_{-}^{\prime}(\lambda) \leq \Delta b^{T} y^{+},
$$

where $y^{+}$denotes the limit of $y^{+}(\epsilon)$ when $\epsilon \downarrow 0$. Since, $f$ is continuous, we conclude that $\left(u^{+}, y^{+}, s^{+}\right)$ is optimal in $\left(\mathrm{QD}_{\lambda}\right)$. Thus, it is feasible in (13). From (14) we conclude that $y^{+}$should be optimal in (13), but $y$ was already an optimal solution of (13). Thus, $\Delta b^{T} y^{+}=\Delta b^{T} y$ which completes the proof. For (12) an analogous argument holds.

It remains to deal with the case where $\lambda$ is an extreme point of dom $(f)$. One may easily verify that if $\lambda$ is the left extreme point of $\operatorname{dom}(f)$ then we can repeat the above arguments. Thus it remains to prove the theorem if $\lambda$ is the right extreme point of $\operatorname{dom}(f)$. Since $f_{+}^{\prime}(\lambda)=\infty$ in that case, we need to show that the above maximization problem (13) is unbounded.

Suppose that (13) is not unbounded. Then the problem and its dual have optimal solutions. The dual problem is given by

$$
\begin{aligned}
\min _{\xi, \eta, \delta, \gamma}\left\{c^{T} \xi+(b+\lambda \Delta b)^{T} \eta:\right. & A \xi=\Delta b, \xi+\gamma x^{*} \geq 0 \\
& \left.A^{T} \eta-Q \xi+\delta s^{*} \geq 0\right\} .
\end{aligned}
$$

We conclude that there exists a vector $\xi \in \mathbb{R}^{n}$ and a scalar $\lambda$ such that $A \xi=\Delta b, Q \xi=0, \xi+\lambda x^{*} \geq 0$. This implies that we cannot have $\xi_{i}<0$ and $x_{i}^{*}=0$. In other words,

$$
x_{i}^{*}=0 \Rightarrow \xi_{i} \geq 0 .
$$

As a consequence, there exists a positive $\varepsilon$ such that $\bar{x}:=x^{*}+\varepsilon \xi \geq 0$. Now we have

$$
A \bar{x}=A\left(x^{*}+\varepsilon \xi\right)=A x^{*}+\varepsilon A \xi=b+(\lambda+\varepsilon) \Delta b .
$$

Thus we find that $\left(Q P_{\lambda+\varepsilon}\right)$ admits $\bar{x}$ as a feasible point. This contradicts the assumption that $\lambda$ is the right extreme point of $\operatorname{dom}(f)$. We conclude that (13) is unbounded, proving the theorem.

Corollary 51 Let $\lambda \in \operatorname{dom}(f)$ belong to the curvy-linearity interval $\left(\lambda_{1}, \lambda_{2}\right)$. Then one has

$$
f^{\prime}(\lambda)=\Delta b^{T} y, \quad \forall \lambda \in\left(\lambda_{1}, \lambda_{2}\right), \forall(x, y, s) \in \mathcal{Q} \mathcal{D}_{\lambda}^{*}, x \in \mathcal{Q P} \mathcal{P}_{\lambda}^{*} .
$$

It is important to note here that the dual optimal set changes. In LP this set is independent of $\lambda$. The next theorem states that on a curvy-linearity interval, a convex combination of two maximal complementary solutions is a maximal complementary solution on this interval again. In LP we have a stronger result; the dual optimal set remains constant on a linearity interval.

Theorem 52 Let $\lambda_{1}, \lambda_{2} \in \operatorname{dom}(f)$. Furthermore, let $\left(x^{(1)}, y^{(1)}, s^{(1)}\right)$ and $\left(x^{(2)}, y^{(2)}, s^{(2)}\right)$ denote the corresponding maximal complementary solutions of $\left(\mathrm{QP}_{\lambda_{1}}\right),\left(\mathrm{QD}_{\lambda_{1}}\right)$ and $\left(\mathrm{QP}_{\lambda_{2}}\right)\left(\mathrm{QD}_{\lambda_{2}}\right)$ respectively. Define for $\lambda \in\left[\lambda_{1}, \lambda_{2}\right]$

$$
\begin{aligned}
& x(\lambda):=(1-\lambda) x^{(1)}+\lambda x^{(2)} \\
& y(\lambda):=(1-\lambda) y^{(1)}+\lambda y^{(2)} \\
& s(\lambda):=(1-\lambda) s^{(1)}+\lambda s^{(2)}
\end{aligned}
$$

Then, the parameters $\lambda_{1}, \lambda_{2}$ belong to the same curvy-linearity interval if and only $x(\lambda), y(\lambda), s(\lambda)$ ) are maximal complementary and optimal solutions of $\left(\mathrm{QP}_{\lambda}\right)$ and $\left(\mathrm{QD}_{\lambda}\right)$. 
Proof: $\quad[\Rightarrow]$ : Let $0<\lambda_{1}, \lambda_{2}<1$ be arbitrary. Consider $\left(x\left(\lambda_{1}\right), y\left(\lambda_{1}\right), s\left(\lambda_{1}\right)\right)$ and $\left(x\left(\lambda_{2}\right), y\left(\lambda_{2}\right), s\left(\lambda_{2}\right)\right)$. We want to show that $\pi_{\lambda_{1}}=\pi_{\lambda_{2}}$. To this end, suppose $x_{i}\left(\lambda_{1}\right)=0$, this means that $\left(x^{(1)}\right)_{i}=\left(x^{(2)}\right)_{i}=$ 0 . So $x_{i}\left(\lambda_{2}\right)=0$. Thus $N_{\lambda_{1}} \cup T_{\lambda_{1}}=N_{\lambda_{2}} \cup T_{\lambda_{2}}$, which indicates that $B_{\lambda_{1}}=B_{\lambda_{2}}$. Now suppose that $s_{k}\left(\lambda_{1}\right)=0$, which means that $\left(s^{(1)}\right)_{k}=\left(s^{(2)}\right)_{k}=0$. Thus, $s_{k}\left(\lambda_{2}\right)=0$. So, $B_{\lambda_{1}} \cup T_{\lambda_{1}}=B_{\lambda_{2}} \cup T_{\lambda_{2}}$. Hence, $N_{\lambda_{1}}=N_{\lambda_{2}}$. Combining these results, we must also have $T_{\lambda_{1}}=T_{\lambda_{2}}$, which completes the proof.

$[\Leftarrow]:$ See the proof of Theorem 40 .

The next lemma (cf. Lemma 22-24) shows a property of strictly complementary solutions of (12), that is needed later on.

Lemma 53 Let $\lambda$ be a transition-point of $f$ and let $\lambda^{+}$belong to the open curvy-linearity interval just to the right of $\lambda$ and $\lambda^{-}$to the open curvy-linearity interval just to the left of $\lambda$. Let $\left(x^{*}, y^{*}, s^{*}\right)$ be a strictly complementary solution of (13) at $\lambda$. Then we have

$$
\begin{aligned}
& \sigma\left(x^{*}\right) \subseteq B_{\lambda^{+}}, \sigma\left(s^{*}\right) \subseteq N_{\lambda^{+}} \\
& \sigma\left(x^{*}\right) \subseteq B_{\lambda^{-}}, \sigma\left(s^{*}\right) \subseteq N_{\lambda^{-}}
\end{aligned}
$$

Proof: We prove only the first part, the second part goes analogously. Without loss of generalization we assume that $\lambda=0$ and that the corresponding optimal partition is given by partition $\pi_{\lambda}=\left(B_{\lambda}, N_{\lambda}, T_{\lambda}\right)$; further assume that the curvy-linearity interval to the right of zero contains $\lambda^{+}=1$ with partition $\pi_{\lambda^{+}}=\left(B_{\lambda^{+}}, N_{\lambda^{+}}, T_{\lambda^{+}}\right)$.

On $(0,1]$ the partition is constant, hence convex combinations of maximal complementary solutions for two values in this interval are optimal in between these two values (cf. (2)). Taking limit to zero implies the existence of $(\underline{x}, \underline{y}, \underline{s})$, optimal in $\left(\mathrm{QP}_{\lambda}\right)$ and $\left(\mathrm{QD}_{\lambda}\right)$, with $\sigma(\underline{x}) \subseteq B_{\lambda^{+}}, \sigma(\underline{s}) \subseteq N_{\lambda^{+}}$ and $\Delta b^{T} \underline{y}=f_{+}^{\prime}(0)=\Delta b^{T} y^{*}$. Since $\left(x^{*}, y^{*}, s^{*}\right)$ and $(\underline{x}, \underline{y}, \underline{s})$ are both optimal in $\left(\mathrm{QP}_{\lambda}\right)$ and $\left(\mathrm{QD}_{\lambda}\right)$, Lemma 4 implies

$$
\begin{aligned}
\left(x^{*}\right)^{T} s^{\left(\lambda^{+}\right)} & =\left(x^{*}\right)^{T}\left(c+Q x^{\left(\lambda^{+}\right)}-A^{T} y^{\left(\lambda^{+}\right)}\right) \\
& =c^{T} \underline{x}+\underline{x}^{T} Q x^{\left(\lambda^{+}\right)}-\underline{x}^{T} A^{T} y^{\left(\lambda^{+}\right)} \\
& =\underline{x}^{T} s^{\left(\lambda^{+}\right)}=0,
\end{aligned}
$$

and

$$
\begin{aligned}
\left(x^{\left(\lambda^{+}\right)}\right)^{T} s^{*} & =\left(x^{\left(\lambda^{+}\right)}\right)^{T}\left(c+Q x^{*}-A^{T} y^{*}\right) \\
& =\left(x^{\left(\lambda^{+}\right)}\right)^{T}(c+Q \underline{x})-(b+\Delta b)^{T} y^{*} \\
& =\left(x^{\left(\lambda^{+}\right)}\right)^{T}(c+Q \underline{x})-(b+\Delta b)^{T} \underline{y} \\
& =\left(x^{\left(\lambda^{+}\right)}\right)^{T}\left(c+Q \underline{x}-A^{T} \underline{y}\right) \\
& =\left(x^{\left(\lambda^{+}\right)}\right)^{T} \underline{s}=0 .
\end{aligned}
$$

Consider now for $\epsilon \in(0,1)$

$$
\begin{aligned}
& x(\varepsilon)=(1-\varepsilon) x^{*}+\varepsilon x^{\left(\lambda^{+}\right)}, \\
& y(\varepsilon)=(1-\varepsilon) y^{*}+\varepsilon y^{\left(\lambda^{+}\right)}, \\
& s(\varepsilon)=(1-\varepsilon) s^{*}+\varepsilon s^{\left(\lambda^{+}\right)},
\end{aligned}
$$

then $x(\varepsilon)$ and $s(\varepsilon)$ are feasible and complementary in $\left(\mathrm{QP}_{\varepsilon}\right)$ and $\left(\mathrm{QD}_{\varepsilon}\right)$, hence optimal. So it holds $\sigma\left(x^{*}\right) \subseteq B_{\lambda+}$ and $\sigma\left(s^{*}\right) \subseteq N_{\lambda+}$. The second part can be proven analogously. 
Note that the inclusions of Lemma 53 hold everywhere. From Theorem 50 we easily obtain the following corollary.

Corollary 54 Let $\lambda$ be a transition-point of $f$. Let $\left(x^{*}, y^{*}, s^{*}\right)$ be a strictly complementary solution of (13) at $\lambda$. Then we have

$$
\sigma\left(x^{*}\right) \subseteq B_{\lambda}, \sigma\left(s^{*}\right) \subseteq N_{\lambda}
$$

An analogous result holds for any strictly complementary solution of (12). The proofs of the results in Section 6.4.3, concerning the inclusions for the optimal sets, follow from the observation that the support of the solutions $\left(x^{*}, s^{*}\right)$ in Lemma 53 and Corollary 54 are equal to the partition on the curvy-linearity interval just to the right of $\lambda$. So, in Lemma 53 we have equality for LP, and Corollary 54 states the same as Corollary 21. The additional results we have in LP, do not hold for QP.

The picture becomes more complete now. Note that Theorem 50 is valid for any value of $\lambda$ in the domain of $f$. The theorem re-establishes that at a 'non-transition' point, where the left and right derivative of $f$ are equal, the value of $\Delta b^{T} y$ is constant when $y$ runs through the optimal sets $\mathcal{Q D}{ }_{\lambda}^{*}$ and $\mathcal{Q P} \mathcal{P}_{\lambda}^{*}$. But it also makes clear that at a transition-point, where the two derivatives might be different, $\Delta b^{T} y$ is not constant when $y$ runs through the optimal sets. Then the extreme values of $\Delta b^{T} y$ yield the left and the right derivative of $f$ at $\lambda$; the left derivative is the minimum and the right derivative the maximal value of $\Delta b^{T} y$ when $y$ runs through the optimal sets. Note that in LP, the solution $y$ corresponding to the maximal value of $\Delta b^{T} y$ is optimal on the linearity interval just to the right of $\lambda$, whereas the solution $y$ corresponding to the minimum value of $\Delta b^{T} y$ is optimal on the linearity interval just to the left of $\lambda$. Therefore, these solutions $y$ are necessarily different and hence the left and right derivatives of $f$ differ in a transition-point. In QP, the solutions $y$ corresponding to the maximal and minimum value of $\Delta b^{T} y$ are not necessarily different, and hence the left and the right derivative of $f$ may be equal in a transition-point. The next theorem states that the transition-points of the optimal value function occur exactly where the first or second order derivative does not exist.

Theorem 55 The transition-points of the optimal value function $f(\lambda)$ occur exactly where its first or second order derivative does not exist.

Proof: $\quad$ Let $\lambda$ denote a transition-point of $f$. Without loss of generality, we assume that $\lambda=0$. Moreover, let $\lambda^{+}=1$ belong to the curvy-linearity interval just to the right of $\lambda$ and let $\lambda^{-}=$ -1 belong to the curvy-linearity interval just to the left of $\lambda$. Suppose to the contrary that the optimal value function is quadratic on $[-1,1]$, while the partition is $\pi_{0}$ for $\lambda=0, \pi_{-1}$ on $[-1,0)$ and $\pi_{1}$ on $(0,1]$. Since the optimal value function is differentiable in $\lambda=0$, it follows from the proof of Lemma 53 that $x(\varepsilon):=(1-\varepsilon) x^{(-1)}+\varepsilon x^{(0)}$ is optimal in $\left(\mathrm{QP}_{\varepsilon-1}\right)$ for $\varepsilon \in(0,1)$, while $x(\varepsilon):=(1-\varepsilon) x^{(0)}+\varepsilon x^{(-1)}$ is optimal in $\left(\mathrm{QP}_{\varepsilon}\right)$ for $\varepsilon \in(0,1)$. Since the derivative of the optimal value function is linear on $[-1,1]$ it follows that $\left(x^{(-1)}+x^{(1)}\right) / 2$ is optimal in $\left(\mathrm{QP}_{l} l_{1}\right)$. This implies $B_{1} \cup B_{-1} \subseteq B_{0}$. Similarly one shows $N_{1} \cup N_{-1} \subseteq N_{0}$. Combining this result with Lemma 53 it follows $B_{1}=B_{-1}=B_{0}$ and $N_{1}=N_{-1}=N_{0}$ contradicting the assumption. This implies the theorem.

As as consequence of Theorem 55 we obtain the analogue of Theorem 25, which can be stated without proof.

Theorem 56 If $f(\lambda)$ is quadratic on the interval $\left[\lambda_{1}, \lambda_{2}\right]$, where $\lambda_{1}<\lambda_{2}$, then the optimal partition $\pi_{\lambda}$ is constant for $\lambda \in\left(\lambda_{1}, \lambda_{2}\right)$.

The next example illustrates that $f$ is not continuously differentiable in general. 


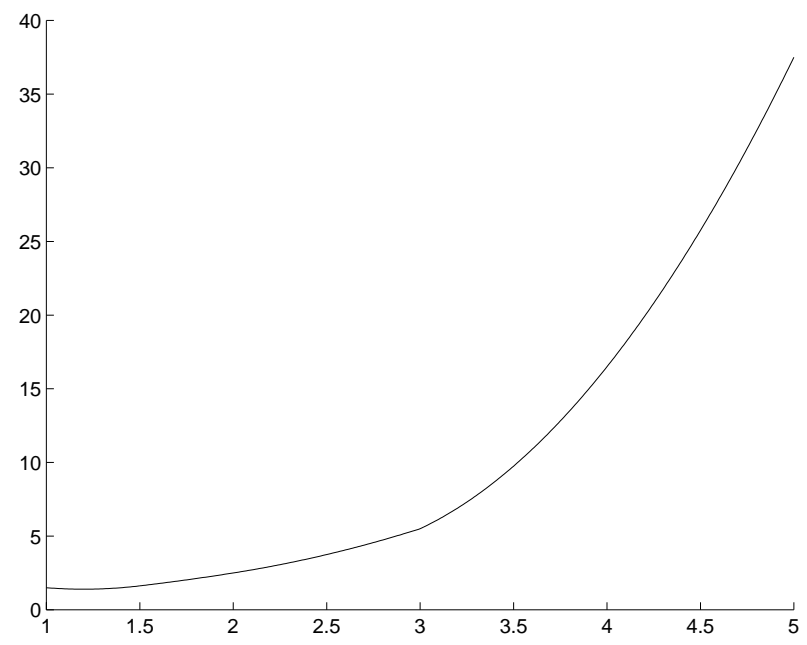

Fig. 2: Non-differentiable value function

Example 3 Consider the parametric QP model with data $c=0$,

$$
Q=\left[\begin{array}{ccc}
3 & 3 & -1 \\
3 & 11 & 23 \\
-1 & 23 & 75
\end{array}\right], A=\left[\begin{array}{lll}
1 & 1 & 1 \\
1 & 3 & 5
\end{array}\right], b=\left(\begin{array}{l}
1 \\
0
\end{array}\right), \Delta b=\left(\begin{array}{l}
0 \\
1
\end{array}\right) \text {. }
$$

At $\lambda=3$ the primal optimal solution is unique, whereas the dual optimal solution is not unique.

\begin{tabular}{c|cccc} 
& $B$ & $N$ & $T$ & $f(\lambda)$ \\
\hline $3<\lambda<5$ & $\{2,3\}$ & $\{1\}$ & $\phi$ & $32 \frac{1}{2}-24 \lambda+5 \lambda^{2}$ \\
$2<\lambda<3$ & $\{1,2\}$ & $\{3\}$ & $\phi$ & $2 \frac{1}{2}-2 \lambda+\lambda^{2}$ \\
$\frac{3}{2}<\lambda<2$ & $\{1,2,3\}$ & $\phi$ & $\phi$ & $\frac{1}{2}+\frac{1}{2} \lambda^{2}$ \\
$1<\lambda<\frac{3}{2}$ & $\{1,3\}$ & $\{2\}$ & $\phi$ & $5-6 \lambda+2 \frac{1}{2} \lambda^{2}$
\end{tabular}

The reader can verify that the optimal value function is not continuously differentiable at $\lambda=3$. Figure 2 illustrates this.

Remark 2 It is interesting to consider the partition $\pi_{\lambda}$ when $\lambda$ runs from $-\infty$ to $\infty$. At the first transition-point, the sets $B_{\lambda}$ and $N_{\lambda}$ increase to a larger set, and when passing to the next open curvy-linearity interval the sets $B_{\lambda}$ and $N_{\lambda}$ become equal to a subset of these enlarged sets. This process repeats itself at every new transition-point: at a transition-point of $f$ the sets $B_{\lambda}$ and $N_{\lambda}$ expand themselves and when passing to the next open curvy-linearity interval they shrink to subsets of the enlarged sets. Since the derivative of $f$ is monotonically increasing when $\lambda$ runs from $-\infty$ to $\infty$ every new set $B_{\lambda}$ and every new set $N_{\lambda}$ arising in this way differs from all previous ones. In other words, every transition-point of $f$ and every curvy-linearity interval of $f$ has its own partition.

To obtain the partition in a neighbouring curvy-linearity interval we have to solve an auxiliary selfdual QP problem. This is contrary to the LP case, where the partition follows as a byproduct of computing the left and right derivatives (see Theorem 19, and Theorem 27). In the next theorem this auxiliary self-dual problem is formulated. 
Theorem 57 Consider the situation as in Lemma 53. Let $\left(x^{*}, s^{*}\right)$ belong to a strictly complementary solution of (13) for $\lambda=0$, and define $B:=\sigma\left(x^{*}\right), N:=\sigma\left(s^{*}\right), T:=\{1, \ldots, n\} \backslash(B \cup N)$. Consider

$$
\begin{aligned}
& \min _{x, s, y}\left\{-\Delta b^{T} y+x^{T} Q x \quad: \quad A x=\Delta b, x^{T} s^{*}=0, x_{T} \geq 0,\right. \\
& \left.A^{T} y+s-Q x=0, s^{T} x^{*}=0, s_{T} \geq 0\right\}
\end{aligned}
$$

and let $(\tilde{x}, \tilde{y}, \tilde{s})$ be a maximal complementary solution of (15). Then $B_{1}=B \cup\left\{i: x_{i}^{*}>0\right\}$, $N_{1}=N \cup\left\{i: s_{i}^{*}>0\right\}$, and $T_{1}=\{1, \ldots, n\} \backslash\left(B_{1} \cup N_{1}\right)$.

Proof: It is easy to check that for a feasible solution of (15)

$$
-\Delta b^{T} y+x^{T} Q x=x^{T}\left(Q x-A^{T} y\right)=x^{T} s=x_{T}^{T} s_{T} \geq 0 .
$$

The dual of (15) is given by

$$
\begin{aligned}
\max _{\eta, x, \sigma, \xi}\left\{\Delta b^{T} \eta-x^{T} Q x: \quad\right. & A \xi=\Delta b, \xi_{N}=0, \xi_{T} \geq 0 \\
& \left.A^{T} \eta+\sigma+Q \xi-2 Q x=0, \sigma_{B}=0, \sigma_{T} \geq 0,\right\},
\end{aligned}
$$

and for a feasible solution it holds

$$
\Delta b^{T} \eta-x^{T} Q x=\eta^{T} A \xi-x^{T} Q x=-\xi^{T} \sigma-(\zeta-x)^{T} Q(\xi-x) \leq 0 .
$$

Consequently, the optimal value of (15), if it exists, is zero. Consider the assignment

$$
x=\xi=x^{(1)}-x^{*}, s=\sigma=s^{(1)}-s^{*}, y=\eta=y^{(1)}-y^{*},
$$

which satisfies the first two linear equalities in (15). Using the fact that $B \subseteq B_{1}$ and $N \subseteq N_{1}$ (Lemma 53) it follows

$$
x_{N}=x_{N}^{(1)}-x_{N}^{*}=0, \quad x_{T}=x_{T}^{(1)}-x_{T}^{*}=x_{T}^{(1)} \geq 0,
$$

and

$$
s_{B}=s_{B}^{(1)}-s_{B}^{*}=0, \quad s_{T}=s_{T}^{(1)}-s_{T}^{*}=s_{T}^{(1)} \geq 0,
$$

and so $x_{T}^{T} s_{T}=0$, implying that the assignment is an optimal solution. The fact that $\left(x^{(1)}, s^{(1)}\right)$ is maximal complementary implies that the assignment must be maximal complementary in (15) as well, implying the result.

We state the dual analogies of the above results without further proof. The omitted proofs are straightforward modifications of the above proofs.

Theorem 58 Let $\mu \in \operatorname{dom}(g)$ and let $\left(x^{*}, y^{*}, s^{*}\right)$ be any optimal solution of $\left(\mathrm{QD}_{\mu}\right)$ and let $x^{*}$ be any optimal solution of $\left(\mathrm{QP}_{\mu}\right)$. Then, the derivatives at $\mu$ satisfy

$$
\begin{aligned}
g_{-}^{\prime}(\mu)=\min _{x, y, s}\left\{\Delta c^{T} x:\right. & A^{T} y+s-Q x=c+\mu \Delta c \\
& s \geq 0, s^{T} x^{*}=0, s_{T}=0, \\
& A x=b, x \geq 0 \\
& \left.x^{T} s^{*}=0, x_{T}=0\right\}, \\
g_{+}^{\prime}(\mu)=\max _{x, y, s}\left\{\Delta c^{T} x:\right. & A^{T} y+s-Q x=c+\mu \Delta c \\
& s \geq 0, s^{T} x^{*}=0, s_{T}=0, \\
& A x=b, x \geq 0 \\
& \left.x^{T} s^{*}=0, x_{T}=0\right\} .
\end{aligned}
$$


Corollary 59 Let $\mu \in \operatorname{dom}(g)$ belong to the curvy-linearity interval $\left(\mu_{1}, \mu_{2}\right)$. Then one has

$$
g^{\prime}(\mu)=\Delta c^{T} x, \quad \forall \mu \in\left(\mu_{1}, \mu_{2}\right), \forall x \in \mathcal{Q} \mathcal{P}_{\mu}^{*},(x, y, s) \in \mathcal{Q D}_{\mu}^{*}
$$

Theorem 60 Let $\mu_{1}, \mu_{2} \in \operatorname{dom}(g)$. Furthermore, let $\left(x^{(1)}, y^{(1)}, s^{(1)}\right)$ and $\left(x^{(2)}, y^{(2)}, s^{(2)}\right)$ denote the corresponding maximal complementary solutions of $\left(\mathrm{QP}_{\mu_{1}}\right),\left(\mathrm{QD}_{\mu_{1}}\right)$ and $\left(\mathrm{QP}_{\mu_{2}}\right),\left(\mathrm{QD}_{\mu_{2}}\right)$ respectively. Define for $\mu \in\left[\mu_{1}, \mu_{2}\right]$

$$
\begin{aligned}
& x(\mu):=(1-\mu) x^{(1)}+\mu x^{(2)} \\
& y(\mu):=(1-\mu) y^{(1)}+\mu y^{(2)} \\
& s(\mu):=(1-\mu) s^{(1)}+\mu s^{(2)} .
\end{aligned}
$$

Then, the parameters $\mu_{1}, \mu_{2}$ belong to the same curvy-linearity interval if and only if $(x(\mu), y(\mu), s(\mu))$ are maximal complementary and optimal solutions of $\left(\mathrm{QP}_{\mu}\right)$ and $\left(\mathrm{QD}_{\mu}\right)$.

Lemma 61 Let $\mu$ be a transition-point of $g$ and let $\mu^{+}$belong to the open curvy-linearity interval just to the right of $\mu$ and $\mu^{-}$to the open curvy-linearity interval just to the left of $\mu$. Let $\left(x^{*}, y^{*}, s^{*}\right)$ be a strictly complementary solution of (17) at $\mu$. Then we have

$$
\begin{aligned}
& \sigma\left(x^{*}\right) \subseteq B_{\mu^{+}}, \sigma\left(s^{*}\right) \subseteq N_{\mu^{+}} \\
& \sigma\left(x^{*}\right) \subseteq B_{\mu^{-}}, \sigma\left(s^{*}\right) \subseteq N_{\mu^{-}}
\end{aligned}
$$

Corollary 62 Let $\mu$ be a transition-point of $g$. Let $\left(x^{*}, y^{*}, s^{*}\right)$ be a strictly complementary solution of (17) at $\mu$. Then we have

$$
\sigma\left(x^{*}\right) \subseteq B_{\mu}, \sigma\left(s^{*}\right) \subseteq N_{\mu}
$$

Theorem 63 If $g(\mu)$ is quadratic on the interval $\left[\mu_{1}, \mu_{2}\right]$, where $\mu_{1}<\mu_{2}$, then the optimal partition $\pi_{\mu}$ is constant for $\mu \in\left(\mu_{1}, \mu_{2}\right)$.

For $g(\mu)$ we have an additional corollary, which easily follows from Theorem 58 . When $Q$ is positive definite, it is easy to show that $x$ is unique. Hence $g(\mu)$ is continuously differentiable.

Corollary 64 Let $Q$ be positive definite, then $g(\mu)$ is continuously differentiable.

Note that Corollary 64 results from the fact that the primal and dual QP problems are not symmetric. Positive definiteness of $Q$ is not a necessary condition for $g(\mu)$ to be continuously differentiable.

Theorem 65 Consider the situation as in Lemma 61 . Let $\left(x^{*}, s^{*}\right)$ be a strictly complementary solution of (17) for $\lambda=0$, and define $B:=\sigma\left(x^{*}\right), N:=\sigma\left(s^{*}\right), T:=\{1, \ldots, n\} \backslash(B \cup N)$. Consider

$$
\begin{aligned}
\min _{x, y, s}\left\{\Delta c^{T} x+x^{T} Q x:\right. & A x=0, x^{T} s^{*}=0, x_{T} \geq 0, \\
& \left.A^{T} y+s-Q x=\Delta c, s^{T} x^{*}=0, s_{T} \geq 0\right\},
\end{aligned}
$$

and let $(\tilde{x}, \tilde{y}, \tilde{s})$ be a maximal complementary solution. Then, $B_{1}=B \cup\left\{i: \tilde{x}_{i}>0\right\}, N_{1}=N \cup\{i$ : $\left.\tilde{s}_{i}^{*}>0\right\}$ and $T_{1}=\{1, \ldots, n\} \backslash\left(B_{1} \cup N_{1}\right)$.

\subsection{Computing the optimal value function}

Using the results of the previous sections, we present in this section an algorithm which yields the optimal value function for a one-dimensional perturbation of the vector $b$ or the vector $c$. We first deal with a one-dimensional perturbation of the vector $b$ with a scalar multiple of the vector $\Delta b$; we state the algorithm for the calculation of the optimal value function and that the algorithm finds all the transition-points and curvy-linearity intervals of it. Having done this it is clear how to treat 


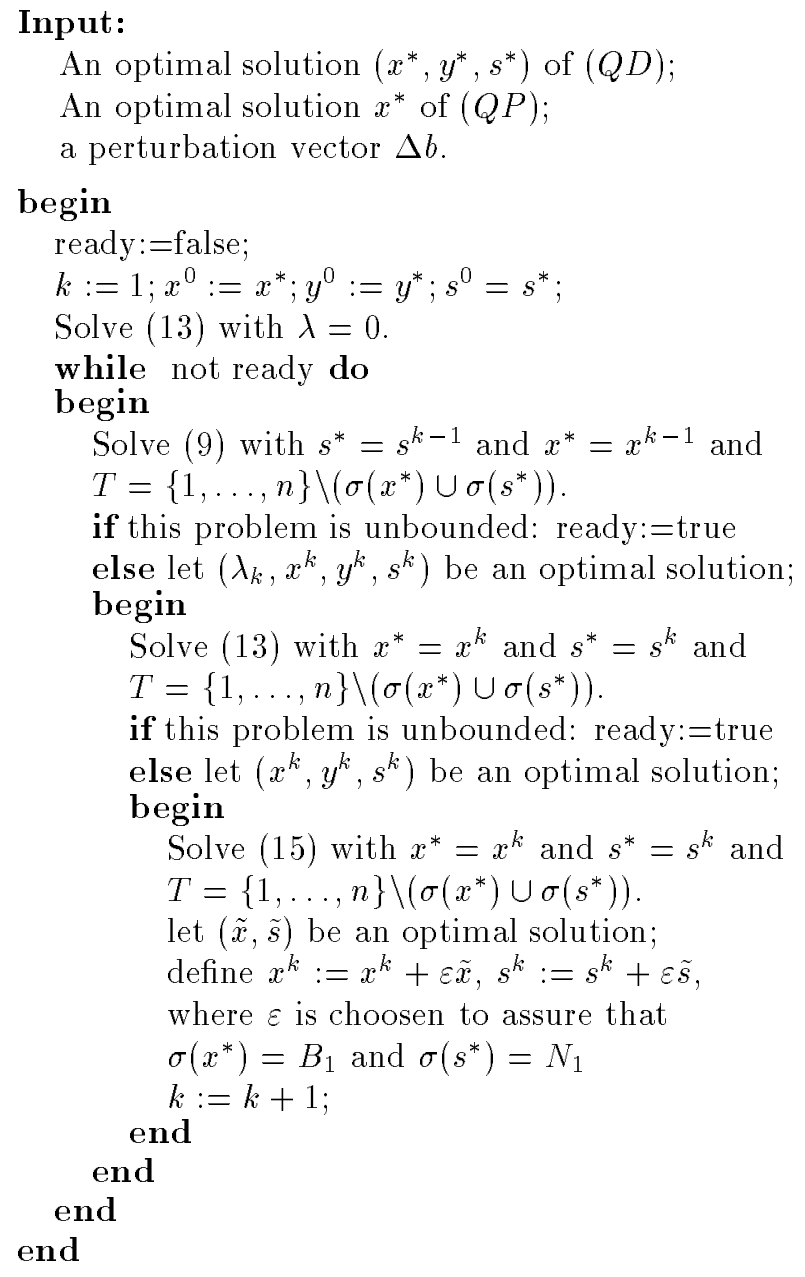

Algorithm 3: The Optimal Value Function $f(\lambda), \lambda \geq 0$

a one-dimensional perturbation of the vector $c$; we also state the corresponding algorithm and its convergence results.

Assume that we have given optimal solutions $x^{*}$ of $(Q P)$ and $\left(x^{*}, y^{*}, s^{*}\right)$ of $(Q D)$. Using the notation of the previous sections, the problems $\left(\mathrm{QP}_{\lambda}\right)$ and its dual $\left(\mathrm{QD}_{\lambda}\right)$ arise by replacing the vector $b$ by $b(\lambda)=b+\lambda \Delta b$; the optimal value of these problems is denoted as $f(\lambda)$. So we have $f(0)=c^{T} x^{*}+\frac{1}{2} x^{* T} Q x^{*}=b^{T} y^{*}-\frac{1}{2} x^{* T} Q x^{*}$. The domain of the optimal value function is $(-\infty, \infty)$ and $f(\lambda)=\infty$ if and only if $\left(Q D_{\lambda}\right)$ is unbounded. Recall from Theorem 43 that $f(\lambda)$ is convex and piecewise quadratic. In Algorithm 3 we present the algorithm which determines $f$ on the nonnegative part of the real line.

The following theorem states that Algorithm 3 finds the successive transition-points of $f$ on the nonnegative part of real line, as well as the slopes of $f$ on the successive curvy-linearity intervals.

Theorem 66 Algorithm 3 terminates after a finite number of iterations. If $K$ is the number of iterations upon termination, then $\lambda_{1}, \lambda_{2}, \cdots, \lambda_{K}$ are the successive transition-points of $f$ on the nonnegative real line. The optimal value at $\lambda_{k}(1 \leq k \leq K)$ is given by $c^{T} x^{k}+\frac{1}{2}\left(x^{k}\right)^{T} Q x^{k}$, and the 
second-order derivative of $f$ on the interval $\left(\lambda_{k}, \lambda_{k+1}\right)(1 \leq k<K)$ by $\frac{\Delta b^{T} \Delta y}{\Delta \lambda}$.

Proof: In the first iteration the algorithm starts by solving 9 where $s^{0}$ is the slack vector in the given optimal solution $\left(x^{0}, y^{0}, s^{0}\right)=\left(x^{*}, y^{*}, s^{*}\right)$ of $(Q D)=\left(Q D_{0}\right)$. This problem is feasible, because $(Q P)$ and $(Q D)$ have an optimal solution $\left(x^{*}, y^{*}, s^{*}\right)$ and $(\lambda, x, y, s)=\left(0, x^{*}, y^{*}, s^{*}\right)$ satisfies the constraints. So the first auxiliary problem is either unbounded or it has an optimal solution $\left(\lambda_{1}, x^{1}, y^{1}, s^{1}\right)$. By Theorem $48 \lambda_{1}$ is equal to the extreme point at the right of the curvy-linearity interval containing 0 . If the problem is unbounded (when $\lambda_{1}=\infty$ ) then $f$ is quadratic on $(0, \infty)$ and the algorithm stops; otherwise $\lambda_{1}$ is the first transition-point right from 0 . (Note that it may happen that $\lambda_{1}=0$. This certainly occurs if 0 is a transition-point of $f$ and the starting solution $\left(x^{*}, y^{*}, s^{*}\right)$ is maximal complementary.) Clearly $x^{1}$ is primal feasible at $\lambda=\lambda_{1}$. Since $\left(x^{1}, y^{1}, s^{1}\right)$ is dual feasible at $\lambda=\lambda_{1}$ and $\left(x^{1}\right)^{T} s^{1}=0$ we see that $x^{1}$ is optimal for $\left(Q P_{\lambda_{1}}\right)$. Hence $f\left(\lambda_{1}\right)=c^{T} x^{1}+\frac{1}{2}\left(x^{1}\right)^{T} Q x^{1}$. Also observe that $\left(x^{1}, y^{1}, s^{1}\right)$ is dual optimal at $\lambda_{1}$. (This also follows from Corollary 21.)

Assuming that the second case occurs, when the above problem has an optimal solution, the algorithm proceeds by solving a second auxiliary problem (13). By Theorem 50 the maximal value is equal to the derivative of $f$ at the right of $\lambda_{1}$. If the problem is unbounded then $\lambda_{1}$ is the largest transition-point of $f$ on $(0, \infty)$ and $f(\lambda)=\infty$ for $\lambda>\lambda_{1}$. In that case we are done and the algorithm stops. In the other case, when the problem is bounded, the optimal solution $\left(x^{1}, y^{1}, s^{1}\right)$ is such that $\Delta b^{T} y^{1}$ is equal to the right derivative of $f$, by Theorem 50 . The second derivative follows from the explicit formula for the optimal value function. To obtain the correct input for the next iteration we need to solve (15). This selfdual QP produces the partition on the neighbouring curvy-linearity interval. Thus we can start the second iteration and proceed as in the first iteration. Since each iteration produces a curvy-linearity interval, and $f$ has only finitely many of such intervals, the algorithm terminates after a finite number of iterations.

The part of the optimal value function to the left of $\lambda_{1}$ can be computed analogously. With respect to the complexity of the algorithm we mention that each subproblem can be solved in polynomial time with an interior point algorithm. However, even in LP there can be exponentially many transition-points (Murty [23]).

When perturbing the vector $c$ with a scalar multiple of $\Delta c$ to $c(\mu)=c+\mu \Delta c$ the algorithm for the calculation of the optimal value function $g(\mu)$ can be stated as in Algorithm 4 (recall that $g$ is concave).

Algorithm 4 finds the successive transition-points of $g$ on the nonnegative real line as well as the slopes of $g$ on the successive curvy-linearity intervals. The proof uses similar arguments as the proof of Theorem 66 and is therefore omitted.

Theorem 67 The algorithm terminates after a finite number of iterations. If $K$ is the number of iterations upon termination, then $\mu_{1}, \mu_{2}, \cdots, \mu_{K}$ are the successive transition-points of $g$ on the nonnegative real line. The optimal value at $\mu_{k}(1 \leq k \leq K)$ is given by $b^{T} y^{k}-\frac{1}{2}\left(x^{k}\right) Q x^{k}$, and the second order derivative of $g$ on the interval $\left(\mu_{k}, \mu_{k+1}\right)(1 \leq k<K)$ by $\frac{\Delta c^{T} \Delta x}{\Delta \lambda}$.

\section{Single Element Perturbation}

Sensitivity analysis (see also Gal [9]) is a special case of parametric programming, where we consider only local information, i.e. ranges and shadow prices on one specific linearity or curvy-linearity interval. In this section we will consider single element perturbation which is also a special case of parametric analysis, where only one element of $b$, or $c$, is perturbed (see, e.g., Gal [9]). So, the perturbation vector is a unit vector. The derivative of the optimal value function to an element of $b$ is called the shadow price and the corresponding linearity interval the range of the element of $b$. 


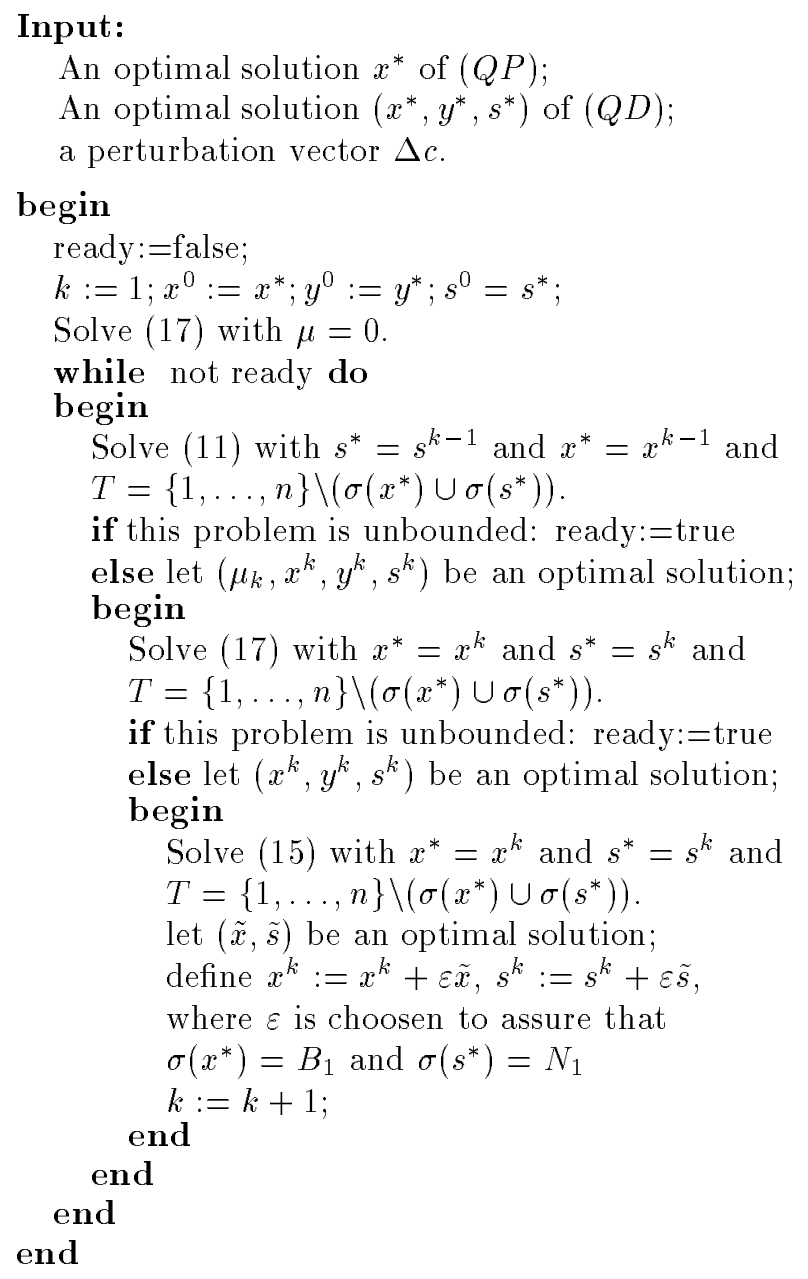

Algorithm 4: The Optimal Value Function $g(\mu), \mu \geq 0$ 
When dealing with single element perturbations the aim is to find the shadow prices and ranges of all elements in $b$ and $c$. The current value of an element of $b$ may be either a transition-point or not. In the latter case the element of $b$ belongs to an open linearity interval and the range of the element of $b$ is this closed linearity interval and the corresponding shadow price is the slope of the optimal value function on this interval. Returning to our example in Section 6.2, we note that the ranges resulting from the classical approach (optimal bases) are subranges of the corresponding linearity intervals. Hence, on linearity intervals the optimal partition remains constant, whereas the optimal basis may change on such intervals.

If there is a transition-point, then we have two shadow prices, the left shadow price, which is the left derivative of the optimal value function at the current value, and the right shadow price, the right derivative of the optimal value function at the current value. In this section we briefly outline how the results in this chapter can be used to perform single element perturbation for LP. The single element perturbation for $\mathrm{QP}$ is left to the reader.

\subsection{Ranges and shadow prices}

Let $x^{*}$ be an optimal solution of $(P)$ and $\left(y^{*}, s^{*}\right)$ an optimal solution of $(D)$. Denoting $e_{i}$ as the $i$-th unit vector $(1 \leq i \leq m)$, the range of element $b_{i}$ of $b$ is simply the linearity interval of the optimal value function $z\left(b+\lambda e_{i}, c\right)$ which contains zero. Using Theorem 15, the extreme points of this linearity interval follow by minimizing and maximizing $\lambda$ over the set

$$
\left\{\lambda: A x=b+\lambda e_{i}, x \geq 0, x^{T} s^{*}=0\right\} .
$$

Considering element $b_{i}$ of $b$ as a variable, the range of $b_{i}$ follows by minimizing and maximizing $b_{i}$ over the set

$$
\left\{b_{i}: A x=b, x \geq 0, x^{T} s^{*}=0\right\} .
$$

For the shadow prices of $b_{i}$ we use Theorem 19. The left and the right shadow price of $b_{i}$ follow by minimizing and maximizing respectively $e_{i}^{T} y=y_{i}$ over the set

$$
\left\{y_{i}: A^{T} y+s=c, s \geq 0, s^{T} x^{*}=0\right\} .
$$

Similarly, the range of element $c_{j}$ of $c$ is equal to the linearity interval of the optimal value function $z\left(b, c+\mu e_{j}\right)$ which contains zero. Changing $c_{j}$ into a variable, and using Theorem 17 the extreme points of this linearity interval follow by minimizing and maximizing $c_{j}$ over the set

$$
\left\{c_{j}: A^{T} y+s=c, s \geq 0, s^{T} x^{*}=0\right\}
$$

For the shadow prices of $c_{j}$ we use Theorem 27. The left and the right shadow price of $c_{j}$ follow by minimizing and maximizing respectively $e_{j}^{T} x=x_{j}$ over the set

$$
\left\{x_{j}: A x=b, x \geq 0, x^{T} s^{*}=0\right\} .
$$

Let us notice the following. If $b_{i}$ is not a transition-point, which becomes evident if the extreme values in (19) both differ from $b_{i}$, then we know that the left and right shadow prices of $b_{i}$ are the same and these are given by $y_{i}^{*}$. So, in that case there is no need to solve (20). On the other hand, when $b_{i}$ is a transition-point, then it is clear from the discussion following Theorem 25 that there are three possibilities. When determining the range of $b_{i}$ by solving (19) the result may be one of the two linearity intervals surrounding $b_{i}$; in that case $y_{i}^{*}$ is the shadow price of $b_{i}$ on this interval. This happens if and only if the given optimal solution $y^{*}$ is such that $y_{i}^{*}$ is an extreme value in the set (20). The third possibility is that the extreme values in the set (19) are both equal to $b_{i}$. This certainly occurs if $y^{*}$ is a strictly complementary solution of $(D)$. In each of the three cases it becomes clear, that after having solved (19), $b_{i}$ is a transition-point. The left and right shadow prices at $b_{i}$ can be found by determining the extreme values of (20). It is clear that similar remarks apply to the range and shadow prices of the elements of the vector $c$. 


\subsection{Using strictly complementary solutions}

The formulas for the ranges and the shadow prices of the elements of $b$ and $c$ can be simplified when the given optimal solutions $x^{*}$ of $(P)$ and $\left(y^{*}, s^{*}\right)$ of $(D)$ are strictly complementary. Let $(B, N)$ denote the optimal partition of $(P)$ and $(D)$. Then we have $x_{B}^{*}>0, x_{N}^{*}=0$ and $s_{B}^{*}=0, s_{N}^{*}>0$. As a consequence, we have $x^{T} s^{*}=0$ in (19) and (22) if and only if $x_{N}=0$. Similarly, $s^{T} x^{*}=0$ holds in (20) and (21) if and only if $s_{B}=0$.

Using this we can reformulate (19) and (20) as

$$
\begin{gathered}
\left\{b_{i}: A x=b, x_{B} \geq 0, x_{N}=0\right\}, \\
\left\{y_{i}: A^{T} y+s=c, s_{B}=0, s_{N} \geq 0\right\} .
\end{gathered}
$$

Similarly, (21) and (22) can be rewritten as

$$
\begin{gathered}
\left\{c_{j}: A^{T} y+s=c, s_{B}=0, s_{N} \geq 0\right\}, \\
\left\{x_{j}: A x=b, x_{B} \geq 0, x_{N}=0\right\} .
\end{gathered}
$$

\section{Summary and Conclusions}

Up till now sensitivity analysis and parametric programming for LP and QP were biased by Simplexbased algorithms. The uprise of interior point methods in the last ten years made it necessary to reconsider the theory of sensitivity analysis and parametric programming. The classical theory is mainly based on the use of an optimal basis. It is well known that optimal bases lead to some major drawbacks in the case of degeneracy of the problem (see Ward and Wendell [28]). This chapter offers an approach which overcomes such difficulties and in which any primal-dual optimal solution can be used to perform a parametric analysis for LP. Our results are based on the property that either the primal or the dual optimal set remains constant when the objective vector or the RHSvector is varied. The presented algorithms can be used in combination with any method for solving linear programs. In the QP-case the so-called optimal partition of the problem is needed in the analysis. In this situation interior point methods provide the natural framework for computations and implementations, since interior point methods generate such a partition (in the limit).

We characterize the optimal value function of parametric linear and parametric quadratic programs in terms of certain segments (linearity resp. curvy-linearity intervals) and transition-points. We show how these segments relate to intervals on which the optimal set and the optimal partition (in linear programs) or the optimal partition (in quadratic programs) remains constant. Moreover algorithms are presented for computing these segments and the optimal value function on these segments. The presented algorithms are new and show promising results as preliminary computations and experiments in MATLAB ${ }^{6}$ have pointed out.

\section{REFERENCES}

[1] Adler, I., R. Monteiro: A geometric view of parametric linear programming, Algorithmica $8(1992) 161-176$.

[2] Anstreicher, K.M., D. Hertog, C. Roos and T. Terlaky: A long step barrier method for convex quadratic programming, Algorithmica 10(1993)365-382.

[3] Bereanu, D.: A property of convex piecewise linear functions with applications to mathematical programming, Unternehmensforschung 9(1965)112-119.

\footnotetext{
${ }^{6}$ The MathWorks Inc., Version 4.2
} 
[4] Berkelaar, A.B., B. Jansen, C. Roos and T. Terlaky: Sensitivity analysis for degenerate quadratic programming. Report 9611/A, Econometric Institute, Erasmus University Rotterdam, P.O. Box 1738, 3000 DR Rotterdam, The Netherlands 1996.

[5] Carpenter, T .J., I .J. Lustig, J .M. Mulvey and D.F. Shanno: Higher order predictor-corrector interior point methods with application to quadratic objectives, SIAM Journal on Optimization $3(1993) 696-725$.

[6] Charnes, A., W. Cooper: Systems evaluation and repricing theorems, Management Science 9(1962)209-228.

[7] Dinkelbach, W.: Sensitivitätsanalysen und parametrische programmierung. Springer-Verlag, New York, USA 1969.

[8] Dorn, W.: Duality in quadratic programming, Quarterly of Applied Mathematics 18(1960)155162.

[9] Gal, T.: Postoptimal analyses, parametric programming and related topics. W. de Gruyter, New York, 2nd edition 1995.

[10] Goldman, A., A. Tucker: "Theory of linear programming." In Kuhn, H., A. Tucker (eds.), Linear Inequalities and Related Systems, Annals of Mathematical Studies Number 38, 53-97. Princeton University Press, Princeton, New Jersey 1956.

[11] Greenberg, H.: An analysis of degeneracy, Naval Research Logistics Quarterly 33(1986)635-655.

[12] Güler, O., Y. Ye: Interior point algorithms for LP generate strictly complementary solutions. Talk given at the ORSA/TIMS Joint National Meeting in Nashville, Tennessee, USA, Dept. of Management Science, University of Iowa, Iowa City, IA 52242, USA 1991.

[13] Güler, O., Y. Ye: Convergence behavior of interior-point algorithms, Mathematical Programming 60(1993)215-228.

[14] Hertog, D.: Interior Point Approach to Linear, Quadratic and Convex Programming. volume 277 of Mathematics and its Applications, Kluwer Academic Publishers, Dordrecht, The Netherlands 1994.

[15] Jansen, B.: Interior Point Techniques in Optimization. Complexity, Sensitivity and Algorithms. PhD thesis, Faculty of Mathematics and Informatics, TU Delft, NL-2628 BL Delft, The Netherlands 1995.

[16] Jansen, B., C. Roos and T. Terlaky: An interior point approach to postoptimal and parametric analysis in linear programming. Technical Report 92-21, Faculty of Technical Mathematics and Informatics, TU Delft, NL-2628 CD Delft, The Netherlands 1992.

[17] Karmarkar, N.K.: A new polynomial-time algorithm for linear programming, Combinatorica 4(1984)373-395.

[18] Kelly, J.: Parametric programming and the primal-dual algorithm, Operations Research $7(1959) 327-334$.

[19] Kriens, J., J. van Lieshout: Notes on the markowitz portfolio selection method, Statistica Neerlandica 42(1988)181-191.

[20] Markowitz, H.: The optimization of a quadratic function subject to linear constraints, Naval Research Logistics Quarterly 3(1956)111-133.

[21] McLinden, L.: The analogue of Moreau's proximation theorem, with applications to the nonlinear complementarity problem, Pacific Journal of Mathematics 88(1980)101-161. 
[22] Mehrotra, S., R.D.C. Monteiro: Parametric and range analysis for interior point methods. Technical Report, Dept. of Systems and Industrial Engineering, University of Arizona, Tucson, AZ 85721, USA 1992.

[23] Murty, K.: Computational complexity of parametric linear programming, Mathematical Programming 19(1980)213-219.

[24] Rubin, D., H. Wagner: Shadow prices: tips and traps for managers and instructors, Interfaces $20(1990) 150-157$.

[25] Vanderbei, R .J.: LOQO: An interior point code for quadratic programming. Technical Report, Dept. of Civil Engineering and Operations Research, Princeton University, Princeton, NJ 08544, USA 1995.

[26] Vörös, J.: Portfolio analysis: an analytic derivation of the efficient portfolio frontier, European Journal of Operations Research 23(1986)294-300.

[27] Vörös, J.: The explicit derivation of the efficient portfolio frontier in the case of degeneracy and general singularity, European Journal of Operations Research 32(1987)302-310.

[28] Ward, J., R. Wendell: Approaches to sensitivity analysis in linear programming, Annals of Operations Research 27(1990)3-38. 